\title{
UNIVALENT MAJORANTS
}

BY

RAPHAEL M. ROBINSON

By $g(z)<G(z)$ for $|z|<R$, we shall mean that $g(0)=G(0)$, that for $|z|<R$ both functions are regular and $G(z)$ is univalent, and that the set of values assumed by $g(z)$ for $|z|<R$ is included in the set of values assumed there by $G(z)$. We say that $G(z)$ is a majorant of $g(z)\left({ }^{1}\right)$. A similar definition may be given with $|z| \leqq R$ in place of $|z|<R$.

We note first that if $G(z)$ is regular and univalent for $|z|<R$, then $g(z)<G(z)$ for $|z|<R$ is equivalent to the existence of a function $\phi(z)$, regular for $|z|<R$, with $g(z)=G(\phi(z))$ and $|\phi(z)| \leqq|z|$. For from $g(z)<G(z)$ it follows that $\phi(z)=G^{-1}(g(z))$ is regular, $|\phi(z)|<R$ for $|z|<R$ and $\phi(0)=0$; hence by Schwarz's lemma, $|\phi(z)| \leqq|z|$. The converse is clear. A like result holds for a closed circle $|z| \leqq R$. Thus if $g(z)<G(z)$ holds in any circle about the origin, it holds also in any smaller such circle.

From the representation $g(z)=G(\phi(z))$ follows also Lindelöf's principle: If $G(z)$ is regular and univalent for $|z|<R$, and if $\left|z_{0}\right|<R$, then the set of possible values of $g\left(z_{0}\right)$ for all functions $g(z)$ with $g(z)<G(z)$ for $|z|<R$ is exactly the map of $|z| \leqq\left|z_{0}\right|$ by $G(z)$. As an example, consider the majorization $g(z)$ $<(1+z) /(1-z)$ for $|z|<1$. By Lindelöf's principle, if $z_{0}$ is a given point in the unit circle, with $\left|z_{0}\right|=r$, then the possible values of $g\left(z_{0}\right)$ fill the circle having the segment from $(1-r) /(1+r)$ to $(1+r) /(1-r)$ as a diameter.

When a majorization $g(z)<G(z)$ for $|z|<R$ is deduced from some hy: pothesis, it will be referred to as sharp, if for every $z_{0}$ with $\left|z_{0}\right|<R, g\left(z_{0}\right)$ can assume for admissible functions $g(z)$ all values permitted by Lindelöf's principle, that is, all points in the map of $|z| \leqq\left|z_{0}\right|$ by $G(z)$.

Some known proofs are advantageously stated in terms of majorants. Consider a proof given by $\operatorname{Radó}\left({ }^{2}\right)$ of the theorem that if $G(z)$ maps $|z|<R$ on a convex region, then it also maps $|z|<r$ on a convex region, for $r<R$. It follows from the definition of convexity that a necessary and sufficient condi-

Presented to the Society, April 28, 1945 ; received by the editors February 18, 1946.

(1) A somewhat more general definition has been used by J. E. Littlewood and by W. Rogosinski, any meromorphic function being allowed as a majorant. Also, they call $g(z)$ subordinate to $G(z)$. See Littlewood, On inequalities in the theory of functions, Proc. London Math. Soc. (2) vol. 23 (1925) pp. 481-519, and Lectures on the theory of functions, Oxford University Press, 1944, chap. 2; Rogosinski, On the coefficients of subordinate functions, Proc. London Math. Soc. (2) vol. 48 (1943) pp. 48-82, and other papers mentioned there. However, these papers are little related to the present one, and the restriction that the majorant be regular and univalent is convenient for our purposes.

(2) T. Rad6, Bemerkung ïber die konformen Abbildungen konvexer Gebiete, Math. Ann. vol. 102 (1929) pp. 428-429. 
tion that $G(z) \operatorname{map}|z|<R$ convexly is that, for every $s$ and $t$ with $|s| \leqq 1$ and $0 \leqq t \leqq 1$, we have

$$
t G(z)+(1-t) G(s z)<G(z) \quad \text { for }|z|<R .
$$

But then, for given $s$ and $t$, we conclude that this majorization also holds for $|z|<r$, so that $|z|<r$ is also mapped on a convex region. A similar theorem is also true for regions star-shaped with respect to $G(0)$. The proof is obtained by putting $s=0$ in the above argument $\left({ }^{3}\right)$.

In this paper, we shall be concerned mainly with the following question: If

$$
g(z)<G(z) \quad \text { for }|z|<1 \text {, }
$$

for what operators $\&$ and radii $r$ can we conclude that

$$
\mathfrak{R g}(z)<\mathfrak{R}(z)
$$$$
\text { for }|z| \leqq r ?
$$

This problem will be studied by two different methods. In Part I, using the first method, we obtain results when $G(z)$ is one of the three special majorants

$$
\frac{1+z}{1-z}, \frac{1}{(1-z)^{2}}, \log \frac{1+z}{1-z}
$$

and $R$ is any of a wide class of operators. In Part II, by the second method, we make a thorough study of the two special operations $l g(z)=z g^{\prime}(z)$ or $[z g(z)]^{\prime}$, with an arbitrary majorant $G(z)$. The inverses of these two operations are considered briefly.

Parts I and II are completely independent, so that either may be read first. The results of both parts are applied to certain special problems in Part III.

\section{PART I}

1.1. The first method depends on the Poisson integral formula, which we shall use in the following form: If $g(z)$ is regular for $|z| \leqq 1$ and $g(0)$ is real, then for $|z|<1$,

$$
g(z)=\frac{1}{2 \pi} \int_{0}^{2 \pi} \Re g\left(e^{i \theta}\right) \frac{1+z e^{-i \theta}}{1-z e^{-i \theta}} d \theta .
$$

This formula may be verified as follows. Twice the right side is

where

$$
\frac{1}{2 \pi i} \int_{|t|=1}[g(t)+\overline{g(t)}] \frac{t+z}{t-z} \frac{d t}{t}=I+J
$$

$$
I=\frac{1}{2 \pi i} \int_{|t|=1} g(t) \frac{t+z}{t-z} \frac{d t}{t}=2 g(z)-g(0)
$$

(3) Compare S. Takahashi, Bemerkung zu einer Arbeit von Herrn Rado, Jap. J. Math. vol. 7 (1930) pp. 161-162. 
by the residue theorem, and

$$
\bar{J}=\frac{1}{2 \pi i} \int_{|t|=1} g(t) \frac{1+\bar{z} t}{1-\bar{z} t} \frac{d t}{t}=g(0) .
$$

Since $g(0)$ is real, we have $I+J=2 g(z)$.

The Poisson integral is particularly adapted to the study of functions with positive real part. As an illustration, let us use this formula to verify Lindelöf's principle for such a function. That is, suppose that $g(z)<(1+z) /(1-z)$ for $|z|<1$, and show that if $\left|z_{0}\right|=r$, then $g\left(z_{0}\right)$ lies in the map of $|z| \leqq r$ by $w=(1+z) /(1-z)$. Without loss of generality, suppose that $g(z)$ is regular for $|z| \leqq 1$. Then by the Poisson integral, $g\left(z_{0}\right)$ is an average of $\left(1+z_{0} e^{-i \theta}\right) /\left(1-z_{0} e^{-i \theta}\right)$, with a certain positive weight factor; that is, an average of the values of $(1+z) /(1-z)$ for $|z|=r$. Hence $g\left(z_{0}\right)$ lies within the convex hull of the map of $|z|=r$ by $(1+z) /(1-z)$. Since this map is a circle, the convex hull is the circle with its interior, as was to be shown.

A more general argument of a similar type is used later. If the mapping involved is not convex, it is convenient to make use of the concept of a hullmajorant in expressing the result. $B y$

$$
g(z)<\mathfrak{S}\{G(z)\} \quad \text { for }|z|<R,
$$

we shall mean that $g(z)$ and $G(z)$ are regular for $|z|<R$, and that for every $r$ with $0 \leqq r<R$, the set of values of $g(z)$ in $|z| \leqq r$ is contained in the convex hull of the set of values of $G(z)$ in that circle. Obviously, this relation is a consequence of $g(z)<G(z)$. The converse is true only if $G(z)$ is univalent and maps convexly.

If the above hull-majorization is deduced from some hypothesis, it will be referred to as sharp, if for every $z_{0}$ with $\left|z_{0}\right|<R, g\left(z_{0}\right)$ can assume for admissible functions $g(z)$ all values in the convex hull of the map of $|z| \leqq\left|z_{0}\right|$ by $G(z)$.

1.2. We shall be concerned with an operator $\mathfrak{R}$ which when applied to a function $g(z)$ regular for $|z|<1$ gives another such function. The transformed function will be denoted by $\mathfrak{R} g(z)$ or by $\mathfrak{R}_{z} g(z)$. The operator $\mathfrak{l}$ will be called linear if it is

(1) additive: $\mathfrak{R}\{g(z)+h(z)\}=\mathfrak{g}(z)+\mathfrak{R}(z)$,

(2) homogeneous: $\mathfrak{R}\{c g(z)\}=c \mathbb{g}(z)$,

(3) continuous: If $g_{n}(z) \rightarrow g(z)$ then $\mathfrak{R} g_{n}(z) \rightarrow \Re g(z)$.

In (3) convergence is understood to mean uniform convergence in the interior of the unit circle. The product of two linear operators is clearly linear.

We shall now show that a linear operation is commutative with an integration. More exactly, if $g(z, t)$ is continuous for $|z|<1$ and $a \leqq t \leqq b$, and regular in $z$ for each fixed $t$, then for any linear operator $\mathfrak{R}$, we have

$$
\mathfrak{R}_{z} \int_{a}^{b} g(z, t) d t=\int_{a}^{b} \mathfrak{R}_{z} g(z, t) d t .
$$

For if we put $\delta_{n}=(b-a) / n$, then 


$$
\int_{a}^{b} g(z, t) d t=\lim _{n \rightarrow \infty}\left[\delta_{n} \sum_{k=0}^{n-1} g\left(z, a+k \delta_{n}\right)\right] .
$$

The convergence is uniform in the interior of the unit circle. Applying $\&$ to both sides, we may bring the $\mathfrak{l}$ on the right after the $\sum$ by using (3), (2), and (1). The right side is then seen to be equal to the integral of $\mathfrak{R}_{z} g(z, t)$.

We shall be concerned with the behavior of the operator $\mathfrak{l}$ when a change of independent variable is made. A linear operator $\&$ will be said to be of order $n$, if for every function $g(z)$ regular in $|z|<1$, and every $\alpha$ with $0<|\alpha| \leqq 1$, we have

$$
\left[\mathfrak{R}_{\zeta} g(\zeta)\right]_{\zeta=\alpha z}=\alpha^{n \mathfrak{R}_{z} g(\alpha z)} \text {. }
$$

We shall show that if $\mathbb{R}$ is of order $n$ and $\mathbb{R}^{\prime}$ of order $m$, then $\mathbb{R}^{\prime} \mathfrak{R}$ is of order $m+n$. Now since $\mathbb{R}^{\prime}$ is of order $m$, we have

$$
\left[R_{\zeta}^{\prime} h(\zeta)\right]_{\zeta-\alpha z}=\alpha^{m R_{z}^{\prime}} h(\alpha z) .
$$

In this formula, substitute $h(z)=\Re g(z)$, and use the fact that $\mathfrak{R}$ is of order $n$. This gives

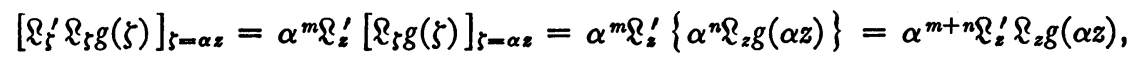

as was to be shown.

We now give some examples of linear operators. Let

$$
\begin{array}{ll}
\mathfrak{P}_{1} g(z)=z g(z), & \mathfrak{Q}_{1} g(z)=z^{-1}[g(z)-g(0)], \\
\mathfrak{P}_{2} g(z)=\int_{0}^{z} g(\zeta) d \zeta, & \mathfrak{Q}_{2} g(z)=g^{\prime}(z) .
\end{array}
$$

It is easily seen that these four operators are linear, and that the $\mathfrak{B}$ 's are of order 1 and the $\mathfrak{Q}$ 's of order -1 . Thus every $\mathfrak{B Q}$ or $\mathfrak{Q} \mathfrak{B}$ is of order zero. Now

$$
\mathfrak{B}_{k} \mathfrak{Q}_{k} g(z)=g(z)-g(0), \quad \mathfrak{Q}_{k} \mathfrak{B}_{k} g(z)=g(z) \quad(k=1,2),
$$

that is, $\mathfrak{B}_{k}$ and $\mathfrak{Q}_{k}$ are essentially inverse. The other combinations give us some interesting examples of linear operators of order zero:

$$
\begin{array}{ll}
\mathfrak{B}_{1} \mathfrak{Q}_{2} g(z)=z g^{\prime}(z), & \mathfrak{Q}_{2} \mathfrak{B}_{1} g(z)=[z g(z)]^{\prime}, \\
\mathfrak{B}_{2} \mathfrak{Q}_{1} g(z)=\int_{0}^{z} \zeta^{-1}[g(\zeta)-g(0)] d \zeta, & \mathfrak{Q}_{1} \mathfrak{P}_{2} g(z)=z^{-1} \int_{0}^{z} g(\zeta) d \zeta .
\end{array}
$$

All of these are used in Part III.

Another linear operator $\mathfrak{R}$ of order zero is obtained by letting $\mathfrak{g} g(z)$ be the $n$th partial sum of the power series expansion of $g(z)$. The various examples which we have given indicate that linear operators of order zero form a rather wide class.

\subsection{Theorem. If}




$$
g(z)<\frac{1+z}{1-z}
$$

for $|z|<1$,

then for any linear operator $\&$ of order zero,

This estimate is sharp( $\left.{ }^{4}\right)$.

$$
\mathfrak{R g}(z)<\mathfrak{S}\left\{\mathfrak{R} \frac{1+z}{1-z}\right\} \quad \text { for }|z|<1 .
$$

Proof. Without loss of generality, suppose $g(z)$ is regular for $|z| \leqq 1$. Applying the linear operator $\mathfrak{R}$ to the Poisson integral ( $\$ 1.1)$ gives

$$
\mathfrak{R}(z)=\frac{1}{2 \pi} \int_{0}^{2 \pi} \Re g\left(e^{i \theta}\right) \mathfrak{R} \frac{1+z e^{-i \theta}}{1-z e^{-i \theta}} d \theta .
$$

Now since $\mathfrak{R}$ is of order zero, this may be written

$$
\Re g(z)=\frac{1}{2 \pi} \int_{0}^{2 \pi} \Re g\left(e^{i \theta}\right)\left[\mathfrak{l}_{\zeta} \frac{1+\zeta}{1-\zeta}\right]_{\zeta=z e^{-i \theta}} d \theta .
$$

Since $\Re g\left(e^{i \theta}\right)$ is positive and has the average 1 , we see that $\mathscr{R} g(z)$ lies within the convex hull of the map of $|\zeta|=|z|$ by $\&_{5}\{(1+\zeta) /(1-\zeta)\}$, as was to be shown. Also, for a given $z$, any point within this convex hull is attained, for $g(z)$ an average of two functions of the form $(1+\alpha z) /(1-\alpha z)$, with $|\alpha|=1$, so that the estimate is sharp.

COROLlaRy. If $G(z)$ maps $|z|<1$ conformally on a half plane, then from

follows

$$
g(z)<G(z) \quad \text { for }|z|<1
$$

$$
\mathfrak{R g}(z)<\mathfrak{S}\{\mathfrak{R} G(z)\} \quad \text { for }|z|<1 \text {, }
$$

for any linear operator $\&$ of order zero. This estimate is sharp.

Proof. We may choose constants $A, B, \alpha$, with $A \neq 0$ and $|\alpha|=1$, such that $A G(\alpha z)+B=(1+z) /(1-z)$. From the hypothesis we find

$$
A g(\alpha z)+B<A G(\alpha z)+B,
$$

hence by the theorem $A \Re_{z} g(\alpha z)+B \Re 1<\mathfrak{g}\left\{A \Re_{z} G(\alpha z)+B \Re_{1}\right\}$. From the fact that $\mathbb{R}$ is of order of zero, it follows that $R 1$ is a constant. Hence $\mathbb{R}_{z} g(\alpha z)<\mathfrak{S}\left\{\mathbb{R}_{z} G(\alpha z)\right\}$, from which the desired conclusion follows.

That this result cannot be true for an arbitrary majorant $G(z)$ is shown by the following counter-examples.

Example 1. Let $G(z)$ be any function which is regular for $|z| \leqq 1$. Consider

(4) Some special cases of this theorem, including $\& g(z)$ equal to a partial sum of the series for $g(z)$, and also $\mathbb{R}=\mathfrak{B}_{2} \mathfrak{Q}_{1}$, have been proved by W. Rogosinski, Über Bildschranken bei Potenzreihen und ihren Abschnitten, Math. Zeit. vol. 17 (1923) pp. 260-276 (Satz II, Satz IV). 
the operator $\mathbb{R}=\mathfrak{B}_{1} \mathfrak{Q}_{2}$ of $\S 1.2$, which is linear and of order zero. From $g(z)<G(z)$ for $|z|<1$, we cannot conclude that $z g^{\prime}(z)<\mathfrak{S}\left\{z G^{\prime}(z)\right\}$ for $|z|<1$. For if so, the boundedness of $g^{\prime}(z)$ would follow from the boundedness of $g(z)$, which is clearly not the case.

Example 2. Start from the majorization $z^{2}<z$ for $|z|<1$, and perform the operation $\mathfrak{R g}(z)=z^{2} g^{\prime \prime}(z)$; here $\mathfrak{l}=\mathfrak{P}_{1}^{2} \mathfrak{Q}_{2}^{2}$, which is linear and of order zero. We obtain $2 z^{2}<\mathfrak{E}\{0\}$, which is not true in any circle.

1.4. We shall be concerned with the average of an analytic function $G(z)$ along a line segment, and shall use for it the notation

$$
\mathfrak{M}\left\{G(z) ; z_{1}, z_{2}\right\}=\frac{1}{z_{2}-z_{1}} \int_{z_{1}}^{z_{2}} G(\zeta) d \zeta,
$$

where the integral is taken along the line segment joining $z_{1}$ and $z_{2}$. We find that

$$
\mathfrak{M}\left\{\frac{1}{(1-z)^{2}} ; z_{1}, z_{2}\right\}=\frac{1}{\left(1-z_{1}\right)\left(1-z_{2}\right)},
$$

provided 1 is not on the segment joining $z_{1}$ and $z_{2}$. That is, the function $1 /(1-z)^{2}$ has the property that its average along a segment is the geometric mean of the values at the end points. This property will be used in studying $1 /(1-z)^{2}$ as a majorant.

We notice first that if $\mathfrak{R}$ is a linear operator of order zero, then

$$
\mathfrak{R}_{z} \mathfrak{M}\{G(\zeta) ; p z,(p+q) z\}=\mathfrak{M}\left\{\mathbb{R}_{5} G(\zeta) ; p z,(p+q) z\right\} .
$$

For if we write the means as real integrals, this becomes

$$
\mathfrak{R}_{z} \int_{0}^{1} G((p+q t) z) d t=\int_{0}^{1}\left[\mathfrak{R}_{5} G(\zeta)\right]_{\zeta=(p+q t) z} d t
$$

and this is seen to be true by first bringing the $\mathfrak{R}$ after the integral sign, and then using the fact that $\mathbb{R}$ is of order zero.

Theorem. If

$$
g(z)<\frac{1}{(1-z)^{2}} \quad \text { for }|z|<1,
$$

then for any linear operator $\mathfrak{R}$ of order zero,

$$
\mathfrak{l g}(z)<\mathfrak{E}\left\{\mathfrak{R} \frac{1}{(1-z)^{2}}\right\} \quad \text { for }|z|<1 .
$$

The result is sharp for $|z| \leqq r$, if the majorant is univalent and convex there, so that the $\mathfrak{S}$ can be omitted.

Proof. We may put 


$$
g(z)=\left(\frac{1+h(z)}{2}\right)^{2}
$$

where

$$
h(z)<\frac{1+z}{1-z} \quad \text { for }|z|>1 .
$$

Without loss of generality, suppose that $h(z)$ is regular for $|z| \leqq 1$. By Poisson's formula,

$$
h(z)=\frac{1}{2 \pi} \int_{0}^{2 \pi} \Re h\left(e^{i \theta}\right) \frac{1+z e^{-i \theta}}{1-z e^{-i \theta}} d \theta,
$$

and hence

$$
g(z)=\left[\frac{1}{2 \pi} \int_{0}^{2 \pi} \Re h\left(e^{i \theta}\right) \frac{1}{1-z e^{-i \theta}} d \theta\right]^{2} .
$$

This may also be written as an iterated integral, which may be put in the form

$$
g(z)=\frac{1}{(2 \pi)^{2}} \int_{0}^{2 \pi} \int_{0}^{2 \pi} \Re h\left(e^{i \lambda}\right) \Re h\left(e^{i \mu}\right) \mathfrak{M}\left\{\frac{1}{(1-\zeta)^{2}} ; z e^{-i \lambda}, z e^{-i \mu}\right\} d \lambda d \mu .
$$

Hence

$$
\mathfrak{R}(z)=\frac{1}{(2 \pi)^{2}} \int_{0}^{2 \pi} \int_{0}^{2 \pi} \Re h\left(e^{i \lambda}\right) \Re h\left(e^{i \mu}\right) \mathfrak{M}\left\{\mathfrak{R} \frac{1}{(1-\zeta)^{2}} ; z e^{-i \lambda}, z e^{-i \mu}\right\} d \lambda d \mu .
$$

On the right, $\mathfrak{R}\left\{1 /(1-\zeta)^{2}\right\}$ is averaged first along a segment joining the two points $z e^{-i \lambda}$ and $z e^{-i \mu}$ on the circle $|\zeta|=|z|$, then this average is averaged with respect to $\lambda$ and $\mu$, a positive weight factor being used. Hence $\& g(z)$ lies in the convex hull of the map of $|\zeta| \leqq|z|$ by $\mathfrak{R}\left\{1 /(1-\zeta)^{2}\right\}$, as was to be shown.

Now $g(z)=1 /(1-\alpha z)^{2}$ is an admissible function, if $|\alpha| \leqq 1$. Hence $\mathbb{R} g\left(z_{0}\right)$ can assume any value in the map of $|z| \leqq\left|z_{0}\right|$ by $\mathfrak{R}\left\{1 /(1-z)^{2}\right\}$. Thus the majorization $\mathfrak{R} g(z) \prec \mathfrak{R}\left\{1 /(1-z)^{2}\right\}$ is sharp for $|z| \leqq r$, if it is correct. By the theorem, it holds if the majorant is univalent and convex there. If $\mathbb{R}$ is the identity, it is true even though the majorant is not convex; and in this case the result of the theorem, with the $\mathfrak{W}$, is certainly not sharp.

Remark. It may be seen that the theorem in general follows from one special case, $\mathfrak{R}=\mathfrak{Q}_{1} \mathfrak{P}_{2}\left({ }^{5}\right)$. For, admitting this case, the hypothesis $g(z)<1 /(1-z)^{2}$

(5) This essential case of the theorem was in effect proved by A. Marx, Untersuchungen ibber schlichte Abbildungen, Math. Ann. vol. 107 (1932) pp. 40-67. For in the proof (pp. 58-61) of Satz C, concerning a convex mapping function $F(z)$, he deduces $F(z) / z<1 /(1-z)$ from $F^{\prime}(z)<1 /(1-z)^{2}$. I have simplified his proof, by making use of the property of $1 /(1-z)^{2}$ stated at the beginning of the section. 
gives

$$
\mathfrak{Q}_{1} \mathfrak{P}_{2} g(z)<1 /(1-z) .
$$

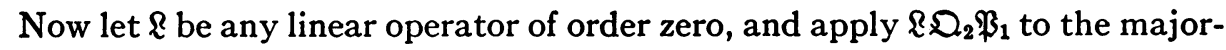
ization just found, as we may by $\$ 1.3$. This gives

$$
\mathfrak{R g}(z)<\mathfrak{S}\left\{\mathfrak{R} \frac{1}{(1-z)^{2}}\right\},
$$

as was to be shown. However, the proof is not simplified by considering the special case.

1.5. In discussing $\log [(1+z) /(1-z)]$ as a majorant, we shall meet the following problem: Suppose that $q(t)$ is a real analytic function for $a \leqq t \leqq b$. What is the maximum possible value of

$$
\int_{a}^{b} p(t) q(t) d t
$$

where $p(t)$ is any function which is piece-wise continuous and for which

$$
-1 \leqq p(t) \leqq 1, \quad \int_{a}^{b} p(t) d t=0 \text { ? }
$$

We notice first that for any real constant $c$,

$$
\int_{a}^{b} p(t) q(t) d t=\int_{a}^{b} p(t)[q(t)-c] d t \leqq \int_{a}^{b}|q(t)-c| d t .
$$

Equality can hold only if

$$
p(t)=\operatorname{sgn}[q(t)-c],
$$

except at a finite number of points. This is an admissible function only for the unique $c$ such that $q(t)$ is more than $c$ in half of the interval of integration, and less in half. For this $c$ and $p(t)$, we both maximize the given integral, and minimize the integral of $|q(t)-c|$.

THEOREM. Let $\mathfrak{R}$ be a linear operator of order 0 . Then for every function $g(z)$ with

we shall also have

$$
g(z)<\log \frac{1+z}{1-z} \quad \text { for }|z|<1,
$$

if and only if the function

$$
\mathfrak{l g}(z)<\mathfrak{l o g} \frac{1+z}{1-z} \quad \text { for }|z| \leqq r,
$$

$$
L(z)=\mathfrak{R} \frac{1+z}{1-z}
$$


has the property that for $|\zeta|=r$, the line joining $L(\zeta)$ and $L(-\zeta)$ does not cross the map of $|z|=r$ by $L(z)$ again.

Furthermore, for any $z_{0}$, the set of possible values of $\& g\left(z_{0}\right)$, for all admissible $g(z)$, is strictly convex (that is, no part of the boundary is a line segment).

Thus the largest possible $r$ for which the first conclusion holds is at least equal to the radius of convexity of $L(z)$ and at most equal to the radius of convexity of the majorant

$$
\mathfrak{l o g} \frac{1+z}{1-z},
$$

and could not be increased by inserting $\mathfrak{W}$ before the right side of the conclusion.

Proof. Suppose for the present that $g(z)$ is regular for $|z| \leqq 1$. If we apply the Poisson integral formula ( $\$ 1.1)$ to $2 g(z) / \pi i$, we find that

$$
g(z)=\frac{i}{4} \int_{0}^{2 \pi} p(\theta) \frac{1+z e^{-i \theta}}{1-z e^{-i \theta}} d \theta,
$$

where

$$
p(\theta)=\Re\left\{2 g\left(e^{i \theta}\right) / \pi i\right\} .
$$

If we apply this formula to $g(z)=\log \left[\left(1+k z e^{-i \beta}\right) /\left(1-k z e^{-i \beta}\right)\right]$, where $k<1$, and then let $k \rightarrow 1$, we find in particular that

$$
\log \frac{1+z e^{-i \beta}}{1-z e^{-i \beta}}=\frac{i}{4} \int_{\beta-\pi}^{\beta+\pi} \operatorname{sgn}(\theta-\beta) \frac{1+z e^{-i \theta}}{1-z e^{-i \theta}} d \theta .
$$

This could also be verified by computing the integral on the right.

The function $p(\theta)$ satisfies the conditions

$$
-1 \leqq p(\theta) \leqq 1, \quad \int_{0}^{2 \pi} p(\theta) d \theta=0 .
$$

Furthermore, if we allow $p(\theta)$ to be any piecewise continuous function satisfying these conditions, then the function $g(z)$ determined by the above integral satisfies the hypothesis of the theorem. A direct verification of this is unnecessary, since it will follow incidentally at a later stage of the proof. We need consider only functions $g(z)$ obtained in this way.

Applying the operator $\&$ to the formula for $g(z)$ gives

Hence

$$
\mathfrak{l g}(z)=(i / 4) \int_{0}^{2 \pi} p(\theta) L\left(z e^{-i \theta}\right) d \theta .
$$

$$
\Re\left\{e^{-i \alpha}\{g(z)\}=(1 / 4) \int_{0}^{2 \pi} p(\theta) \Re\left\{i e^{-i \alpha} L\left(z e^{-i \theta}\right)\right\} d \theta .\right.
$$


We wish to maximize this expression for a fixed $z=z_{0}$ with $\left|z_{0}\right|=r$, and all admissible $p(\theta)$. By the result at the beginning of this section, the sharp bound is given by

$$
\Re\left\{e^{-i \alpha} \mathfrak{R}\left(z_{0}\right)\right\} \leqq(1 / 4) \int_{0}^{2 \pi}\left|\Re\left\{i e^{-i \alpha} L\left(z_{0} e^{-i \theta}\right)\right\}-c(\alpha)\right| d \theta,
$$

and is attained only if

$$
p(\theta)=\operatorname{sgn}\left[\Re\left\{i e^{-i \alpha} L\left(z_{0} e^{-i \theta}\right)\right\}-c(\alpha)\right],
$$

except possibly at a finite number of points, where $c(\alpha)$ is so determined that $p(\theta)$ is 1 in half of the interval of integration and -1 in half.

We thus have to divide the map of the circle $|z|=r$ by the function $L(z)$ by a line of given orientation, in such a way that the part on each side corresponds to arcs of $|z|=r$ amounting to a semicircle. If and only if the line joining $L(\zeta)$ and $L(-\zeta)$ does not cut this curve again, for every $\zeta$ with $|\zeta|=r$, it is true that the dividing line always cuts the curve into just two pieces, each corresponding to half of $|z|=r$, so that $p(\theta)=1$ and $p(\theta)=-1$ each hold on a complete semicircle. In this case, as noticed above, we have for the maximizing function $g_{0}(z)$, with a suitable $\beta$,

$$
g_{0}(z)=\log \frac{1+z e^{-i \beta}}{1-z e^{-i \beta}} .
$$

Thus in this case,

$$
\Re\left\{e^{-i \alpha} \mathfrak{g}\left(z_{0}\right)\right\} \leqq \Re\left\{e^{-i \alpha}\left\{g_{0}\left(z_{0}\right)\right\} \leqq \max _{|z|=r}\left\{\left\{e^{-i \alpha} \mathfrak{l} \log \frac{1+z}{1-z}\right\},\right.\right.
$$

and hence

$$
\mathfrak{g}(z)<\mathfrak{Q}\left\{\mathfrak{R} \log \frac{1+z}{1-z}\right\} \quad \text { for }|z| \leqq r .
$$

If $L(z)$ is such that, for some $\zeta$ with $|\zeta|=r$, the line joining $L(\zeta)$ and $L(-\zeta)$ does cross the map of $|z|=r$ again, then this conclusion is false.

In particular, if we let $\mathfrak{R}$ be the identity, we have

$$
g(z) \prec \log \frac{1+z}{1-z} \quad \text { for }|z|<1,
$$

which verifies the statement made earlier that any $p(\theta)$ satisfying the stated conditions leads to an admissible $g(z)$.

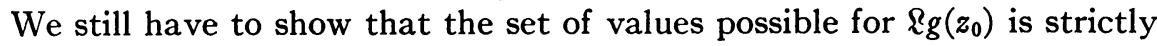
convex. It will then follow that the conclusion with or without $\mathfrak{S}$ is equivalent, and the proof will be complete. It may be remarked that the convexity of the set is clear, since an average of two values of $g\left(z_{0}\right)$ is obtained by starting from an average of the two corresponding functions $p(\theta)$. 
Let $K$ be any closed convex set, and $S$ a closed subset which has at least one point in common with each supporting line of $K$. It is easily seen that if a supporting line of $K$ has a segment in common with $K$, then both end points of this segment must belong to $S$. Hence if $S$ has exactly one point in common with each supporting line of $K$, then $K$ must be strictly convex.

Let us apply this result with $K$ the set of all possible values of $g g\left(z_{0}\right)$, and $S$ the set of values of $R g\left(z_{0}\right)$ corresponding to functions $p(\theta)$ which are always equal to \pm 1 , and which have a bounded number of discontinuities. This set $S$ is closed. Also, if we allow as many discontinuities as the maximum number of times a straight line can cross the map of $|z|=r$ by $L(z)$, then the maximum of $\Re\left\{e^{-i \alpha} \Omega g\left(z_{0}\right)\right\}$, for all $g(z)$, occurs for one and essentially only one such $p(\theta)$. That is, the maximum of $\Re\left\{e^{-i \alpha} w\right\}$, for $w$ in $K$, occurs at exactly one point $w$ in $S$. Hence $K$ is strictly convex, as was to be shown.

\section{PART II}

2.1. Using our second method, we shall study, for certain special operators $\mathbb{R}$, the question whether for a given majorant $G(z)$ and for all $g(z)$,

$$
g(z)<G(z) \quad \text { for }|z|<1
$$

implies

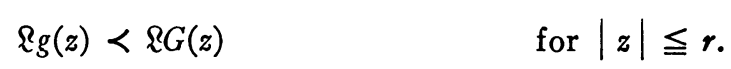

We wish to find the largest $r$ for which this is true for a given $G(z)$.

A brief description of the method will now be given. Let $h(z)=R g(z)$ and $H(z)=\mathscr{R} G(z)$, and suppose that $H(z)$ is univalent for $|z| \leqq r$. To show that $h(z)<H(z)$ for $|z| \leqq r$, we must show, for each $z_{0}$ with $\left|z_{0}\right| \leqq r$, that $h\left(z_{0}\right)$ lies in the map of $|z| \leqq r$ by $H(z)$. It will be sufficient to find a $\zeta_{0}$ with $\left|\zeta_{0}\right| \leqq r$, such that $\left|h\left(z_{0}\right)-H\left(\zeta_{0}\right)\right| \leqq\left|H(Z)-H\left(\zeta_{0}\right)\right|$ for $|Z|=r$. We shall in fact always choose $\zeta_{0}$ so that $g\left(z_{0}\right)=G\left(\zeta_{0}\right)$; it is then perhaps reasonable to suppose that $h\left(z_{0}\right)$ will be near $H\left(\zeta_{0}\right)$.

This method is quite successful for $\mathfrak{R g}(z)=z g^{\prime}(z) \quad(\S \S 2.2-2.4)$ and for $\mathfrak{g}(z)=[z g(z)]^{\prime}(\$ \S 2.5-2.7)$. Conditions are derived from which the largest possible $r$ for a given majorant $G(z)$ may be calculated. Also, the largest common radius for all univalent majorants is found to be $3-2^{3 / 2}$ in the first case, and $1 / 3$ in the second. The method is also applied, but with less success, to the two inverse operations ( $\$ 2.8)$.

2.2. THEOREM. If $G(z)$ is regular and univalent for $|z|<1$, then a necessary and sufficient condition that for every function $g(z)$ with

we shall also have

$$
g(z)<G(z) \quad \text { for }|z|<1
$$

$$
z g^{\prime}(z) \prec z G^{\prime}(z)
$$$$
\text { for }|z| \leqq r
$$

is that $z G^{\prime}(z)$ is univalent for $|z| \leqq r$ and 


$$
\left|\frac{Z G^{\prime}(Z)-\zeta G^{\prime}(\zeta)}{G^{\prime}(\zeta)}\right| \geqq \frac{r^{2}-|\zeta|^{2}}{1-r^{2}} \quad \text { for }|\zeta| \leqq|Z|=r .
$$

Proof. By hypothesis, $g(z)=G(\phi(z))$, where $\phi(z)$ is regular and $|\phi(z)|<1$ for $|z|<1$, and $\phi(0)=0$. We may put $\phi(z)=z \psi(z)$, where $|\psi(z)| \leqq 1$ for $|z|<1$. For the mapping $w=\psi(z)$, we have by the invariant form of Schwarz's lemma,

$$
\frac{|d w|}{1-|w|^{2}} \leqq \frac{|d z|}{1-|z|^{2}}
$$

or

$$
\left|\psi^{\prime}(z)\right| \leqq \frac{1-|\psi(z)|^{2}}{1-|z|^{2}}
$$

and this is the only condition to which $\psi^{\prime}(z)$ is subject for given $z$ and $\psi(z)$. The equivalent inequality for $\phi^{\prime}(z)$ is $\left.{ }^{6}\right)$

$$
\left|z \phi^{\prime}(z)-\phi(z)\right| \leqq \frac{|z|^{2}-|\phi(z)|^{2}}{1-|z|^{2}} .
$$

If we put $\zeta=\phi(z)$, then we find that

$$
z g^{\prime}(z)-\zeta G^{\prime}(\zeta)=G^{\prime}(\zeta)\left[z \phi^{\prime}(z)-\phi(z)\right]
$$

and hence

$$
\left|z g^{\prime}(z)-\zeta G^{\prime}(\zeta)\right| \leqq\left|G^{\prime}(\zeta)\right| \frac{|z|^{2}-|\zeta|^{2}}{1-|z|^{2}}
$$

For a given majorant $G(z)$, this is the only condition imposed on $g^{\prime}(z)$ at a given point $z$ when the corresponding value of $g(z)$ is given; $\zeta$ is to be determined from $g(z)=G(\zeta)$. If we are given only that $|z| \leqq r$, then the only condition to which $z g^{\prime}(z)$ is subject is that it lies in the circle

$$
\left|w-\zeta G^{\prime}(\zeta)\right| \leqq\left|G^{\prime}(\zeta)\right| \frac{r^{2}-|\zeta|^{2}}{1-r^{2}}
$$

for some $\zeta$ with $|\zeta| \leqq r$.

We can draw the desired conclusion if and only if, for every $\zeta$ with $|\zeta| \leqq r$, this circle lies within the map of $|z| \leqq r$ by $z G^{\prime}(z)$. Because of the univalence of $z G^{\prime}(z)$, this is equivalent to

$$
\left|Z G^{\prime}(Z)-\zeta G^{\prime}(\zeta)\right| \geqq\left|G^{\prime}(\zeta)\right| \frac{r^{2}-|\zeta|^{2}}{1-r^{2}} \quad \text { for }|Z|=r
$$

( ${ }^{\circ}$ This inequality was given by J. Dieudonne, who drew from it the interesting conclusion that $\left|\phi^{\prime}(z)\right| \leqq 1$ for $|z| \leqq 2^{1 / 2}-1$. See Recherches sur quelques problemes relatifs aux polynomes et aux fonctions bornées, Ann. Ecole Norm. (3) vol. 48 (1931) pp. 247-358, especially pp. 351-352. 

that

Corollary. A necessary condition to obtain the conclusion of the theorem is

$$
\left|1+\frac{z G^{\prime \prime}(z)}{G^{\prime}(z)}\right| \geqq \frac{2 r}{1-r^{2}} \quad \text { for }|z| \leqq r .
$$

Proof. If we let $\zeta \rightarrow Z$ then

$$
Z G^{\prime}(Z)-\zeta G^{\prime}(\zeta) \sim(Z-\zeta)\left[Z G^{\prime}(Z)\right]^{\prime} .
$$

If the approach is radial, then $|Z-\zeta|=r-|\zeta|$. This leads to the condition

$$
\left|\frac{\left[Z G^{\prime}(Z)\right]^{\prime}}{G^{\prime}(Z)}\right| \geqq \frac{2 r}{1-r^{2}} \quad \text { for }|Z|=r .
$$

Since $z G^{\prime}(z)$ is univalent, its derivative doesn't vanish, so that this condition must hold for $|z| \leqq r$.

Remark. In particular, putting $z=0$ gives $1 \geqq 2 r /\left(1-r^{2}\right)$, or

$$
r \leqq 2^{1 / 2}-1 \text {, }
$$

as a necessary condition for the conclusion of the theorem. For the particular majorant $G(z)=z$, the condition of the theorem becomes

$$
|Z-\zeta| \geqq\left(r^{2}-|\zeta|^{2}\right) /\left(1-r^{2}\right),
$$

which is seen to be true if $1 \geqq 2 r /\left(1-r^{2}\right)$, and hence for the maximum possible $r$. Thus we obtain Dieudonné's theorem: If $|g(z)|<1$ for $|z|<1$, and $g(0)=0$, then $\left|g^{\prime}(z)\right| \leqq 1$ for $|z| \leqq 2^{1 / 2}-1$.

2.3. We ask now whether the necessary condition of the corollary is also sufficient. While this question is not completely answered, we are able to show that it is sufficient if $G(z)$ maps $|z| \leqq r$ on a convex region.

We must first prove a lemma about convex mapping functions. We notice to begin with that if $K$ is a convex region, having the inner radius $R$ with respect to the point $a$, then $K$ contains the circle $|w-a|<R / 2$. For if not, $K$ is included in some half-plane, whose boundary is at a distance $d<R / 2$ from $a$; but the inner radius of this half-plane with respect to $a$ is $2 d<R$, which gives a contradiction.

LEMмA. If $G(z)$ is regular and convex for $|z| \leqq r$, then

$$
\left|\frac{G(Z)-G(\zeta)}{G^{\prime}(\zeta)}\right| \geqq \frac{r^{2}-|\zeta|^{2}}{2 r} \quad \text { for }|\zeta|<|Z|=r .
$$

Proof. The inner radius of $|z|<r$ with respect to $\zeta$ is $r\left(1-|\zeta / r|^{2}\right)$, hence the inner radius of the map of $|z|<r$ by $G(z)$ with respect to $G(\zeta)$ is

$$
\left|G^{\prime}(\zeta)\right| r\left(1-|\zeta / r|^{2}\right) \text {. }
$$

The distance $|G(Z)-G(\zeta)|$ from $G(\zeta)$ to a boundary point must be at least 
half of this, which gives the required inequality.

THEOREM. Suppose that $G(z)$ is regular and univalent for $|z|<1$, and convex for $|z| \leqq r$. Then a necessary and sufficient condition that for every function $g(z)$ with

$$
g(z)<G(z) \quad \text { for }|z|<1
$$

we shall also have

$$
z g^{\prime}(z) \prec z G^{\prime}(z) \quad \text { for }|z| \leqq r
$$

is that

$$
\left|1+\frac{z G^{\prime \prime}(z)}{G^{\prime}(z)}\right| \geqq \frac{2 r}{1-r^{2}} \quad \text { for }|z| \leqq r .
$$

Remark. The condition for convexity is known to be

$$
\Re\left\{1+\frac{z G^{\prime \prime}(z)}{G^{\prime}(z)}\right\} \geqq 0 \quad \text { for }|z| \leqq r .
$$

Thus both the convexity condition and the other condition of the theorem concern the same quantity; the one places it in the right half-plane, the other outside of a certain circle about the origin.

Proof. Since $G(z)$ is convex for $|z| \leqq r, z G^{\prime}(z)$ is univalent, and indeed maps $|z| \leqq r$ on a star-shaped region. Thus to show the sufficiency of our necessary condition, we have only to derive from it and the convexity condition the inequality required in the theorem of $\$ 2.2$. It is sufficient to prove the inequality for the point $Z G^{\prime}(Z)$ nearest to $\zeta G^{\prime}(\zeta)$. Join these two points by a straight line. This segment lies within the map of $|z| \leqq r$ by $z G^{\prime}(z)$, since this function is univalent. It is the transform of a curve $C$ joining $\zeta$ to $Z$. We then have

$$
\begin{aligned}
\left|Z G^{\prime}(Z)-\zeta G^{\prime}(\zeta)\right| & =\int_{C}\left|\left[z G^{\prime}(z)\right]^{\prime}\right||d z| \\
& =\int_{C}\left|G^{\prime}(z)\right|\left|1+\frac{z G^{\prime \prime}(z)}{G^{\prime}(z)}\right||d z| \\
& \geqq \frac{2 r}{1-r^{2}} \int_{C}\left|G^{\prime}(z)\right||d z| \geqq \frac{2 r}{1-r^{2}}|G(Z)-G(\zeta)| .
\end{aligned}
$$

Hence by the lemma

$$
\left|\frac{Z G^{\prime}(Z)-\zeta G^{\prime}(\zeta)}{G^{\prime}(\zeta)}\right| \geqq \frac{2 r}{1-r^{2}} \frac{r^{2}-|\zeta|^{2}}{2 r}=\frac{r^{2}-|\zeta|^{2}}{1-r^{2}}
$$

2.4. TheOREM. If

$$
g(z)<G(z)
$$$$
\text { for }|z|<1 \text {, }
$$ 
then

$$
z g^{\prime}(z)<z G^{\prime}(z) \quad \text { for }|z| \leqq 3-2^{3 / 2}=0.171 \cdots .
$$

The number $3-2^{3 / 2}$ cannot be replaced by any larger constant.

Proof. It is known that for a univalent function $G(z)$, the quantity

$$
w=1+\frac{z G^{\prime \prime}(z)}{G^{\prime}(z)}
$$

is subject, for $|z| \leqq r$, only to the condition that it lie in the $\operatorname{circle}\left({ }^{7}\right)$

$$
\left|w-\frac{1+r^{2}}{1-r^{2}}\right| \leqq \frac{4 r}{1-r^{2}} .
$$

Thus $\Re w \geqq 0$ if $1+r^{2}-4 r \geqq 0$, or $r \leqq 2-3^{1 / 2}=0.267 \cdots$; this is the known radius of convexity for univalent functions. The other condition, $|w|$ $\geqq 2 r /\left(1-r^{2}\right)$, follows only if $1+r^{2}-4 r \geqq 2 r$, or $r \leqq 3-2^{3 / 2}$. This determines the required radius, since the convexity condition holds in a larger circle.

Remark. If $G(z)=z /(1+z)^{2}$, then

$$
1+\frac{z G^{\prime \prime}(z)}{G^{\prime}(z)}=\frac{1-4 z+z^{2}}{1-z^{2}} .
$$

Thus the required inequality holds at $z=r$ only for $r \leqq 3-2^{3 / 2}$, so that $G(z)$ is an extremal function; it is essentially the only one.

We may ask, for what $g(z)$ does the conclusion not hold in any larger circle? There is no such function; on the contrary, the method of proof shows that infinitesimal variations of $G(z)$ are decisive. For any particular $g(z)$, a radius larger than $3-2^{3 / 2}$ may be found.

We shall show now that even if we add the hypothesis that $g(z)$ is. univalent for $|z|<1$, we cannot replace $3-2^{3 / 2}$ by any larger constant. We again consider $G(z)=z /(1+z)^{2}$, and determine $g(z)$ from

$$
\frac{g(z)}{g(z)-1 / 4}=(1-\epsilon) \frac{G(z)}{G(z)-1 / 4},
$$

that is, by making a linear transformation leaving 0 and $1 / 4$ fixed. We find that

$$
g(z)=\frac{(1-\epsilon) z}{(1+z)^{2}-4 \epsilon z} .
$$

(7) This result will be found in any treatment of the classical theory of univalent functions, because of the central position which it occupies in that theory. It bridges the gap between the inequality $\left|a_{2}\right| \leqq 2$ and the bounds for $\left|G^{\prime}(z)\right|$ ("distortion theorem"), for a function $G(z)=z+a_{2} z^{2}+\cdots$, regular and univalent in the unit circle, in that it is obtained from the former by a linear transformation, and leads to the latter by in tegration. 
This function maps $|z|<1$ on the $w$ plane slit from $1 / 4$ to $+\infty$ and from $-(1-\epsilon) / 4 \epsilon$ to $-\infty$. We have evidently $g(z)<G(z)$ for $|z|<1$. Now the conclusion $z g^{\prime}(z)<z G^{\prime}(z)$ for $|z| \leqq r$ cannot hold if we have $g^{\prime}(r)>G^{\prime}(r)$. This condition reduces to

$$
(1-\epsilon)(1+r)^{4}>\left[(1+r)^{2}-4 \epsilon r\right]^{2},
$$

which is true for small $\epsilon$ if $(1+r)^{4}<8 r(1+r)^{2}$, or $r>3-2^{3 / 2}$.

Remark. According to a theorem of Biernacki( $\left.{ }^{8}\right)$, if $g(z)<G(z)$ for $|z|<1$, and if $g^{\prime}(0) / G^{\prime}(0) \geqq 0$, then $|g(z)| \leqq|G(z)|$ at least for $|z| \leqq 1 / 4$. Using our theorem, we see that also $\left|g^{\prime}(z)\right| \leqq\left|G^{\prime}(z)\right|$ at least for $|z| \leqq 0.04$. Such a result was found by Biernacki only under the additional hypothesis that $g(z)$ is univalent.

2.5. The considerations of $\S \S 2.2$ and 2.3 concerning the operation $\mathfrak{g} g(z)$ $=z g^{\prime}(z)$ can be extended almost immediately to the operation $\mathfrak{l} g(z)=[z g(z)]^{\prime}$. The results are formulated in this and the next section. On the other hand, the result analogous to that of $\$ 2.4$ requires a new method, which is set out in $\S 2.7$.

THEOREM. If $G(z)$ is regular and univalent for $|z|<1$, then a necessary and sufficient condition that for every function $g(z)$ with

$$
g(z) \prec G(z)
$$$$
\text { for }|z|<1
$$

we shall also have

$$
[z g(z)]^{\prime} \prec[z G(z)]^{\prime}
$$$$
\text { for }|z| \leqq r
$$

is that $[z G(z)]^{\prime}$ is univalent for $|z| \leqq r$ and

$$
\left|\frac{[Z G(Z)]^{\prime}-[\zeta G(\zeta)]^{\prime}}{G^{\prime}(\zeta)}\right| \geqq \frac{r^{2}-|\zeta|^{2}}{1-r^{2}} \quad \text { for }|\zeta| \leqq|Z|=r .
$$

Proof. This is practically identical with the proof of the theorem of $\$ 2.2$. We need only notice that, with the notation used there, $g(z)=G(\zeta)$, and hence

$$
[z g(z)]^{\prime}-[\zeta G(\zeta)]^{\prime}=z g^{\prime}(z)-\zeta G^{\prime}(\zeta) .
$$

Corollary. A necessary condition to obtain the conclusion of the theorem is that

$$
\left|2+\frac{z G^{\prime \prime}(z)}{G^{\prime}(z)}\right| \geqq \frac{2 r}{1-r^{2}} \quad \text { for }|z| \leqq r .
$$

Proof. By letting $\zeta \rightarrow Z$ radially, we obtain the condition

$$
\left|\frac{[Z G(Z)]^{\prime \prime}}{G^{\prime}(Z)}\right| \geqq \frac{2 r}{1-r^{2}} \quad \text { for }|Z|=r .
$$

(8) M. Biernacki, Sur les fonctions univaientes, Mathematica vol. 12 (1936) pp. 49-64, especially $\$ 2$. 
Since $[z G(z)]^{\prime}$ is univalent, its derivative doesn't vanish, so that this condition must also hold for $|z| \leqq r$.

Remark. In particular, putting $z=0$ gives $2 \geqq 2 r /\left(1-r^{2}\right)$, or

$$
r \leqq\left(5^{1 / 2}-1\right) / 2=0.618 \cdots,
$$

as a necessary condition for the conclusion of the theorem. For the particular majorant $G(z)=z$, the condition of the theorem becomes

$$
|2 Z-2 \zeta| \geqq\left(r^{2}-|\zeta|^{2}\right) /\left(1-r^{2}\right),
$$

which is seen to be true for the maximum possible radius. The result for this case may be written in the following form: If $f(z) / z<z$ for $|z|<1$, then $f^{\prime}(z)<2 z$ for $|z| \leqq\left(5^{1 / 2}-1\right) / 2$; in other words $\left({ }^{9}\right)$, if $f(z)$ is regular and $|f(z)|<1$ for $|z|<1$, and if $f(0)=0$ and $f^{\prime}(0)=0$, then $\left|f^{\prime}(z)\right| \leqq 2|z|$ for $|z| \leqq\left(5^{1 / 2}-1\right) / 2$.

2.6. Theorem. Suppose that $G(z)$ is regular and univalent for $|z|<1$, and convex for $|z| \leqq r$. Then a necessary and sufficient condition that for every function $g(z)$ with

$$
g(z)<G(z)
$$$$
\text { for }|z|<1
$$

we shall also have

$$
[z g(z)]^{\prime}<[z G(z)]^{\prime}
$$

for $|z| \leqq r$

is that $[z G(z)]^{\prime}$ is univalent for $|z| \leqq r$, and

$$
\left|2+\frac{z G^{\prime \prime}(z)}{G^{\prime}(z)}\right| \geqq \frac{2 r}{1-r^{2}}
$$

for $|z| \leqq r$.

Proof. In this case, the univalence of $[z G(z)]^{\prime}$ is assumed, since it is not known to follow from the convexity of $G(z)$. Otherwise, the proof is entirely analogous to that of the theorem of $\$ 2.3$. Here $C$ should be a curve joining $\zeta$ to $Z$, which is transformed by $[z G(z)]^{\prime}$ into a line segment. We have

$$
\begin{aligned}
\left|[Z G(Z)]^{\prime}-[\zeta G(\zeta)]^{\prime}\right| & =\int_{c}\left|[z G(z)]^{\prime \prime}\right||d z| \\
& =\int_{C}\left|G^{\prime}(z)\right|\left|2+\frac{z G^{\prime \prime}(z)}{G^{\prime}(z)}\right||d z| \\
& \geqq \frac{2 r}{1-r^{2}} \int_{C}\left|G^{\prime}(z)\right||d z| \geqq \frac{2 r}{1-r^{2}}|G(Z)-G(\zeta)| .
\end{aligned}
$$

Hence by the lemma of $\S 2.3$, we derive the condition of the theorem of $\S 2.5$.

2.7. The problem to be considered is for what radius we can draw the conclusion $[z g(z)]^{\prime}<[z G(z)]^{\prime}$ for all univalent majorants $G(z)$. We first examine

(9) Compare Robinson, Bounded analytic functions, University of California Publications in Mathematics n.s. vol. 1 (1944) pp. 131-146 (top of p. 142). 
the possibility of proceeding as in $\$ 2.4$; that is, by applying the theorem of $\S 2.6$. The quantity $w=2+z G^{\prime \prime}(z) / G^{\prime}(z)$ lies in the circle

$$
\left|w-\frac{2}{1-r^{2}}\right| \leqq \frac{4 r}{1-r^{2}}
$$

provided $|z| \leqq r$. Hence $|w| \geqq 2 r /\left(1-r^{2}\right)$ provided $2-4 r \geqq 2 r$, or $r \leqq 1 / 3$. The other necessary condition, that $[z G(z)]^{\prime}$ be univalent, will be shown below to hold in a circle of radius more than $1 / 3$. Unfortunately, the auxiliary condition that $G(z)$ be convex may hold only for $|z| \leqq 2-3^{1 / 2}$, so that we cannot determine the sharp radius from $\$ 2.6$. We find only that the desired conclusion can be drawn at least for $|z| \leqq 2-3^{1 / 2}$, and at most for $|z| \leqq 1 / 3$.

Consequently, we are forced to go back to the original (and more complicated) conditions of the theorem of $\$ 2.5$. We shall show first that $[z G(z)]^{\prime}$ is univalent in a circle of radius more than $1 / 3$, and then that the required inequality holds for $r=1 / 3$.

LEMMA. If $G(z)$ is regular and univalent for $|z|<1$, then $H(z)=[z G(z)]^{\prime}$ is univalent at least for $|z| \leqq 0.38$.

Remark. It appears likely that the sharp radius is $1 / 2$. For it is easily seen that $|z|<1 / 2$ is the largest circle in which $H^{\prime}(z) \neq 0$ always holds.

Proof. We shall show not only that $H(z)$ is univalent for $|z| \leqq 0.38$, but also that it maps this circle on a star-shaped region. Since $H^{\prime}(0)=2 G^{\prime}(0) \neq 0$, it will be sufficient to show that

$$
\Re\left\{\frac{z H^{\prime}(z)}{H(z)}\right\} \geqq 0 \quad \text { for }|z| \leqq r
$$

is true with $r=0.38$. We shall write the quantity to be estimated in the form

$$
\frac{z H^{\prime}(z)}{H(z)}=\left[2+\frac{z G^{\prime \prime}(z)}{G^{\prime}(z)}\right]:\left[1+\frac{G(z)}{z G^{\prime}(z)}\right] .
$$

The numerator is restricted to the previously mentioned circle, and hence its amplitude does not exceed arc $\sin 2 r$ numerically. We proceed to estimate the amplitude of the denominator. By a theorem of (irunsky $\left({ }^{10}\right)$, we have

$$
\left|\log \frac{z G^{\prime}(z)}{G(z)}\right| \leqq \log \frac{1+r}{1-r} .
$$

Using the inequality $\left|e^{s}-1\right| \leqq e^{|s|}-1$, with $s=\log \left[G(z) / z G^{\prime}(z)\right]$, we find

$$
\left|\frac{G(z)}{z G^{\prime}(z)}-1\right| \leqq \frac{2 r}{1-r} \text {. }
$$

(10) H. Grunsky, Neue Abschätzungen zur konformen Abbildung, Schriften aus dem Mathematischen Seminar der Universität Bє lin vol. 1 (1932) pp. 95-140 (s ee p. 130). 
Thus the denominator which we are estimating is restricted to the circle $|w-2| \leqq 2 r /(1-r)$, from which $\mid$ amp $w \mid \leqq \operatorname{arc} \sin [r /(1-r)]$ follows. Consequently,

$$
\left|\operatorname{amp} \frac{z H^{\prime}(z)}{H(z)}\right| \leqq \arcsin 2 r+\arcsin \frac{r}{1-r} .
$$

The right side is found to be less than $90^{\circ}$ for $r=0.38$.

TheOREM. If

$$
g(z) \prec G(z)
$$

$$
\text { for }|z|<1
$$

then

$$
[z g(z)]^{\prime}<[z G(z)]^{\prime} \quad \text { for }|z| \leqq 1 / 3 .
$$

The number $1 / 3$ cannot be replaced by any larger constant.

Proof. We have only to show that the inequality of the theorem of $\$ 2.5$ holds for $r=1 / 3$. For each $\zeta$, we need verify this condition only for the point $Z$ on $|Z|=r$ for which $[Z G(Z)]^{\prime}$ is nearest to $[\zeta G(\zeta)]^{\prime}$. The line segment joining these points is the transform of a curve $C$ joining $\zeta$ to $Z$, and lying in $|z| \leqq r$. This is the same choice of $C$ as in $\$ 2.6$, and the equation used there is also needed here. We shall now write it in the form

$$
\begin{aligned}
& \left|\frac{[Z G(Z)]^{\prime}-[\zeta G(\zeta)]^{\prime}}{G^{\prime}(\zeta)}\right| \\
& \quad=\frac{1-|\zeta|^{2}}{2} \int_{C} \frac{\left|G^{\prime}(z)\right|\left(1-|z|^{2}\right)}{\left|G^{\prime}(\zeta)\right|\left(1-|\zeta|^{2}\right)} \cdot\left|2+\frac{z G^{\prime \prime}(z)}{G^{\prime}(z)}\right| \cdot \frac{2|d z|}{1-|z|^{2}} .
\end{aligned}
$$

We have written the integrand on the right as the product of three factors, of which the third is simply the hyperbolic element of distance $d s$. The first factor is the ratio of the magnification by the mapping $w=G(z)$ at two points, $z$ and $\zeta$, when distance in the $z$-plane is measured in the hyperbolic metric. Hence its possible values, for the class of univalent functions, depend only on the hyperbolic distance between $z$ and $\zeta$. On the other hand, the second factor is the absolute value of a quantity which is known to be restricted to the circle stated at the beginning of this section, and hence the sharp lower bound is given by

$$
\left|2+\frac{z G^{\prime \prime}(z)}{G^{\prime}(z)}\right| \geqq \frac{2-4|z|}{1-|z|^{2}} .
$$

It will be sufficient to consider the case $\zeta=\rho>0$. We shall show that in this case, the smallest value of the integral is attained when $\dot{C}$ is the segment $\rho \leqq z \leqq r$, and

$$
G(z)=z /(1+z)^{2} .
$$

To see this, first draw a circle with hyperbolic center $\rho$ and passing through $r$. The curve $C$ must extend from $\rho$ at least to this circle, and throwing away 
the part of $C$ outside the circle tends to decrease the integral. Let us now, for various different paths $C$, compare an element of the integral at a given hyperbolic distance $D$ from $\rho$, less than the hyperbolic radius of the circle. We see that: (1) The possible values of the first factor depend only on $D$. If $z>\rho$, the minimum value is attained for $G(z)=z /(1+z)^{2}$; to see this, we need only make a linear transformation taking $\rho$ to 0 , and then apply the classical distortion theorem. (2) For a given $D$, the maximum $|z|$, and hence the minimum value of the second factor, is attained only for $z>\rho$; and the extremal function in this case is again $G(z)=z /(1+z)^{2}$. (3) The third factor $d s$ is smaller along a hyperbolic radius of the circle than along any other curve; and the segment $\rho \leqq z \leqq r$ is such a radius. From these three statements, the desired result follows.

Hence the condition of the theorem of $\$ 2.5$ will be satisfied for all univalent functions $G(z)$, if it is for the function $G(z)=z /(1+z)^{2}$, with $\zeta=\rho$ and $Z=r$. The condition may be written

$$
\left(1-r^{2}\right)\left\{[r G(r)]^{\prime}-[\rho G(\rho)]^{\prime}\right\} \geqq\left(r^{2}-\rho^{2}\right) G^{\prime}(\rho) \quad \text { for } 0 \leqq \rho \leqq r .
$$

Since this condition is true for $\rho=r$, it is sufficient to verify that, for $0 \leqq \rho \leqq r$, the derivatives of the two sides with respect to $\rho$ satisfy an inequality in the opposite sense:

$$
-\left(1-r^{2}\right)[\rho G(\rho)]^{\prime \prime} \leqq\left(r^{2}-\rho^{2}\right) G^{\prime \prime}(\rho)-2 \rho G^{\prime}(\rho) .
$$

Substituting the value of $G(z)$, this reduces to

$$
\left(1-r^{2}\right)(1-2 \rho) \geqq\left(r^{2}-\rho^{2}\right)(2-\rho)+\rho\left(1-\rho^{2}\right),
$$

or

$$
2 \rho \leqq 1-3 r^{2},
$$

and hence is true for $0 \leqq \rho \leqq r$ provided $2 r \leqq 1-3 r^{2}$, or $r \leqq 1 / 3$.

Remark. A method similar to that used here could also have been used in proving the Theorem of $\$ 2.4$; but in that case we had a simpler method.

2.8. In $\$ \S 2.2-2.4$ we considered the operation $\ell g(z)=z g^{\prime}(z)$, and in $\S \S 2.5-$ 2.7 the operation $g g(z)=[z g(z)]^{\prime}$. For both of these differentiation operations, rather complete results were obtained. In this section, we shall consider briefly the inverse operations. Unfortunately, only rather weak results are proved, though it appears likely that the conclusion of ten holds in the entire unit circle.

THEOREM. If $g(z)$ and $G(z)$ are regular for $|z|<1$,

$$
z g^{\prime}(z)<z G^{\prime}(z) \quad \text { for }|z|<1,
$$

and $g(0)=G(0)$, then

$$
g(z) \prec G(z) \quad \text { for }|z| \leqq r,
$$

provided $r \leqq 2^{1 / 2}-1$ and 


$$
\min _{|z| \leqq r}\left|G^{\prime}(z)\right|: \max _{|z| \leqq r}\left|G^{\prime}(z)\right| \geqq \log \frac{1+r}{1-r},
$$

and hence certainly for $r=1 / 5$.

Proof. We may suppose $G(0)=0$. We must first investigate the univalence of $G(z)$ for $|z| \leqq r$. Since $z G^{\prime}(z)$ is univalent for $|z|<1$, it defines a star mapping at least for $|z| \leqq \tanh \pi / 4=0.655 \cdots$, as was shown by Grunsky $\left(^{11}\right)$, and hence $G(z)$ is univalent and convex in this circle.

It remains to show that the values assumed by $g(z)$ in $|z| \leqq r$ are included in the set of values assumed by $G(z)$. Now by hypothesis, $z g^{\prime}(z)=\phi(z) G^{\prime}(\phi(z))$, where $\phi(z)$ is regular and $|\phi(z)|<1$ for $|z|<1$, and $\phi(0)=0$. Hence

$$
g(z)=\int_{0}^{z} g^{\prime}(t) d t=\int_{0}^{z} \phi(t) t^{-1} G^{\prime}(\phi(t)) d t .
$$

On the other hand,

$$
G(\phi(z))=\int_{0}^{z} G^{\prime}(\phi(t)) \phi^{\prime}(t) d t
$$

Hence

$$
g(z)-G(\phi(z))=\int_{0}^{z}\left[\phi(t)-t \phi^{\prime}(t)\right] t^{-1} G^{\prime}(\phi(t)) d t .
$$

The integral may be taken along a radius. Now

$$
\left|\phi(t)-t \phi^{\prime}(t)\right| \leqq \frac{|t|^{2}-|\phi(t)|^{2}}{1-|t|^{2}}
$$

as was pointed out in $\$ 2.2$. Hence

$$
|g(z)-G(\phi(z))| \leqq \int_{0}^{z}(|t|-|\phi(t)|) \frac{|t|+|\phi(t)|}{|t|}\left|G^{\prime}(\phi(t))\right| \frac{|d t|}{1-|t|^{2}} .
$$

We estimate the various factors of the integrand. By the theorem of Dieudonné (see end of $\$ 2.2$ ), we have $\left|\phi^{\prime}(t)\right| \leqq 1$ for $|t| \leqq 2^{1 / 2}-1$, hence $|t|-|\phi(t)|$ increases along the radius from 0 to $z$ if $|z| \leqq 2^{1 / 2}-1$. In particular,

$$
|t|-|\phi(t)| \leqq|z|-|\phi(z)| \leqq r-|\phi(z)|
$$

for $r \leqq 2^{1 / 2}-1,|z| \leqq r$, and $t$ on the radius joining 0 and $z$. Also,

$$
\frac{|t|+|\phi(t)|}{|t|} \leqq 2, \quad\left|G^{\prime}(\phi(t))\right| \leqq \max _{|\zeta| \leqq r}\left|G^{\prime}(\zeta)\right|
$$

(11) The truth and sharpness of this result follows at once from the inequality of Grunsky quoted in $\$ 2.7$ (see footnote 10), and was stated explicitly in his note, Zwei Bemerkungen zur konformen Abbildung, Jber. Deutschen Math. Verein. vol. 43 (1933) pp. 140-143. 
Putting in these bounds, and integrating the remaining factor, we have

$$
|g(z)-G(\phi(z))| \leqq(r-|\phi(z)|) \max _{|\zeta| \leqq r}\left|G^{\prime}(\zeta)\right| \log \frac{1+r}{1-r} .
$$

On the other hand, we have for $|Z|=r$, by a familiar argument (integration along a curve transformed into a straight line), the inequality

$$
|G(Z)-G(\phi(z))| \geqq \min _{|\zeta| \leqq r}\left|G^{\prime}(\zeta)\right|(r-|\phi(z)|) .
$$

Thus we shall have

$$
|g(z)-G(\phi(z))| \leqq|G(Z)-G(\phi(z))| \quad \text { for }|z| \leqq r,|Z|=r,
$$

and hence $g(z)$ in the map of $|z| \leqq r$ by $G(z)$, provided the inequality stated in the theorem holds.

If we put $R=(1+r) /(1-r)$, this inequality takes the form $\min / \max$ $\geqq \log R$. Now the classical bounds for a univalent function, applied to $z G^{\prime}(z)$, give $\min / \max \geqq 1 / R^{2}$. Hence it is sufficient to have $1 / R^{2} \geqq \log R$, which is true for $R=3 / 2$, or $r=1 / 5$.

- IOREM. If $g(z)$ and $G(z)$ are regular for $|z|<1$, and

$$
[z g(z)]^{\prime}<[z G(z)]^{\prime} \quad \text { for }|z|<1 \text {, }
$$

then

$$
g(z) \prec G(z) \quad \text { at least for }|z| \leqq 1 / 5 .
$$

Proof. From the hypothesis, it follows that $g(0)=G(0)$. We may suppose that $G(0)=0$ and $G^{\prime}(0)>0$. If we put

$$
H(z)=[z G(z)]^{\prime},
$$

then also $H(0)=0$ and $H^{\prime}(0)>0$. By the rotation theorem for univalent functions, in the sharp form found by Golusin( $\left.{ }^{12}\right)$,

$$
\left|\operatorname{amp} H^{\prime}(z)\right| \leqq 4 \arcsin r \quad \text { for }|z| \leqq r,
$$

provided $r \leqq 2^{-1 / 2}$, and hence in particular,

$$
\Re H^{\prime}(z) \geqq 0 \quad \text { for }|z| \leqq \sin \pi / 8=0.382 \cdots \text {. }
$$

We shall show that $G(z)$ is univalent at least for $|z| \leqq \sin \pi / 8$, by showing that $\Re G^{\prime}(z) \geqq 0$. This may be seen from the formula

$$
G^{\prime}(z)=z^{-2} \int_{0}^{z}\left[t^{2} G^{\prime}(t)\right]^{\prime} d t=z^{-2} \int_{0}^{z} H^{\prime}(t) t d t,
$$

(12) G. M. Golusin, Über die Verzerrungssätze der schlichten konformen Abbildungen (Russian with German summary), Rec. Math. (Mat. Sbornik) N.S. vol. 1 (1936) pp. 127-135 (Theorem 6). 
where the integral is taken along a radius, so that $z^{-2} t d t$ is positive.

To show that $g(z)<G(z)$ for $|z| \leqq r$ (where $r \leqq \sin \pi / 8$ ), it remains to show that the set of values assumed by $g(z)$ is included in the set of values assumed by $G(z)$. Now by hypothesis, $[z g(z)]^{\prime}=\phi(z) G^{\prime}(\phi(z))+G(\phi(z))$, where $\phi(z)$ is regular and $|\phi(z)|<1$ for $|z|<1$, and $\phi(0)=0$. Hence

Also

$$
z g(z)=\int_{0}^{z}\left[\phi(t) G^{\prime}(\phi(t))+G(\phi(t))\right] d t .
$$

Thus

$$
z G(\phi(z))=\int_{0}^{z}\left[t G^{\prime}(\phi(t)) \phi^{\prime}(t)+G(\phi(t))\right] d t
$$

$$
g(z)-G(\phi(z))=z^{-1} \int_{0}^{z}\left[\phi(t)-t \phi^{\prime}(t)\right] G^{\prime}(\phi(t)) d t .
$$

This differs from the corresponding formula in the proof of the preceding theorem only in having a factor $t^{-1}$ replaced by $z^{-1}$, which is smaller in absolute value. Thus the estimates made there still apply; in fact, we could make slightly better estimates here. In any case, the remainder of the proof given there applies equally well here.

\section{PART III}

3.1. Suppose that $f(z)$ and $F(z)$ are regular for $|z|<1$, and have the form $z+\cdots$ We shall study the relations between the three majorizations
(A) $\frac{f(z)}{z}<\frac{F(z)}{z}$,
(B) $f^{\prime}(z)<F^{\prime}(\underline{z})$,
(C) $\frac{z f^{\prime}(z)}{f(z)}<\frac{z F^{\prime}(z)}{F(z)}$.

Supposing that one of the majorizations holds for $|z|<1$, we ask in how large a circle $|z| \leqq r$ one of the others must hold; there are six possible combinations. It will be convenient to introduce also the majorization

$$
\log \frac{f(z)}{z}<\log \frac{F(z)}{z} .
$$

The relation between $(A)$ and $\left(A^{\prime}\right)$ is the following: From $(A)$ follows $\left(A^{\prime}\right)$, provided $F(z) / z \neq 0$; from ( $\mathrm{A}^{\prime}$ ) follows (A), provided $F(z) / z$ is univalent, which is true if $F(z) / z$ is never negative.

By applying the linear operator $\mathfrak{R}$ defined by $\mathfrak{g} g(z)=[z g(z)]^{\prime}$ to $(\mathrm{A})$, we obtain (B). Similarly, by applying the operator $\mathfrak{R}$ defined by $\mathbb{R} g(z)=z g^{\prime}(z)$ to $\left(A^{\prime}\right)$, and adding 1 to both sides, we obtain $(C)$. The inverse operations lead from (B) to $(A)$ and from $(C)$ to $\left(A^{\prime}\right)$. I do not know any method to relate $(B)$ and $(C)$, except via (A). This leads to incomplete results.

We shall carry out in detail the method just described only for the case in which the given majorant, $F(z) / z, F^{\prime}(z)$, or $z F^{\prime}(z) / F(z)$, is $(1+z) /(1-z)$. The 
theory in Parts I and II gives here rather complete results. It may be remarked that the present problem was the starting point for this paper, and that the more general results were worked out in an attempt to solve it. The six cases of the problem are covered in the following theorem.

THEOREM. Suppose that $f(z)=z+\cdots$ is regular for $|z|<1$.

If

$$
\frac{f(z)}{z}<\frac{1+z}{1-z}
$$

for $|z|<1$,

then

$$
f^{\prime}(z)<\frac{2}{(1-z)^{2}}-1 \quad \text { for }|z| \leqq 1 / 2
$$

and

(AC) $\frac{z f^{\prime}(z)}{f(z)}<\frac{2 z}{1-z^{2}}+1 \quad$ for $|z| \leqq \frac{1+5^{1 / 2}}{2}-\left(\frac{1+5^{1 / 2}}{2}\right)^{1 / 2}=0.346 \cdots$. If

$$
f^{\prime}(z)<\frac{1+z}{1-z} \quad \text { for }|z|<1 \text {, }
$$

then

$$
\frac{f(z)}{z}<\frac{2}{z} \log \frac{1}{1-z}-1 \quad \text { for }|z|<1
$$

and

$$
\frac{z f^{\prime}(z)}{f(z)} \prec \frac{1+z}{1-z}:\left(\frac{2}{z} \log \frac{1}{1-z}-1\right) \quad \text { at least for }|z| \leqq 0.3 \text {. }
$$

If

$$
\frac{z f^{\prime}(z)}{f(z)}<\frac{1+z}{1-z} \quad \text { for }|z|<1 \text {, }
$$

then

$$
\frac{f(z)}{z}<\frac{1}{(1-z)^{2}}
$$

$$
\text { for }|z|<1 \text {, }
$$

and

$$
f^{\prime}(z)<\frac{1+z}{(1-z)^{3}} \quad \text { at least for }|z| \leqq \frac{5-17^{1 / 2}}{2}=0.438 \cdots
$$


If we replace $\prec$ by $=$ in any one of the three hypotheses, we must make the same replacement in the two corresponding conclusions. Only in the two cases $(\mathrm{BC})$ and $(\mathrm{CB})$ do we not obtain the largest radius in which the conclusion holds. The result (CA), and a weaker form of $(\mathrm{CB})$, were obtained by A. Marx.

The six conclusions of this theorem are proved in $\S \S 3.3-3.8$, respectively. The proofs are independent, except that $(B C)$ depends on $(B A)$, and $(C B)$ on (CA). Conclusions (AB) and (AC) are proved by two methods, using Part I or Part II; (BA) and (CA) are proved by the first method; while $(\mathrm{BC})$ and $(\mathrm{CB})$ are proved by the second method, but using results proved by the first method. Also, (CB) for $|z| \leqq 2-3^{1 / 2}=0.267 \cdots$ is proved by the first method.

In $\$ 3.9$, we show that from (CA) for $|z|<1$ follows (C) only for $|z| \leqq 1 / 3$.

3.2. Before proceeding to the proof of the various parts of our theorem, we have some comments to make concerning its interest and sharpness.

We have been considering the three expressions
(a)
$g_{1}(z)=f(z) / z$,
$g_{2}(z)=f^{\prime}(z)$,
$g_{3}(z)=z f^{\prime}(z) / f(z)$,

where $f(z)=z+\cdots$ is regular for $|z|<1$; when considering (c), we shall suppose that $f(z) \neq 0$ for $z \neq 0$. Notice that each of the three expressions measures in some sense the magnification and rotation accomplished by the mapping $w=f(z)$. The first measures distance and direction from the origin, the second the local scale and orientation, while the third measures the local scale and orientation relative to the distance and direction from the origin.

The hypothesis $g_{k}(z)<(1+z) /(1-z)$, which occurs in the theorem, is equivalent to $\Re g_{k}(z)>0$. This seems a particularly interesting majorization, because it means that in the mapping $w=f(z)$, the rotation (in the relevant sense) is less than a right angle. Furthermore, for $k=2$ or 3 , this condition implies that $f(z)$ is univalent. For if $\Re f^{\prime}(z)>0$, then $\left[f\left(z_{2}\right)-f\left(z_{1}\right)\right] /\left(z_{2}-z_{1}\right)$, which is the average of $f^{\prime}(z)$ on the segment joining $z_{1}$ and $z_{2}$, also has a positive real part; and $\Re\left\{z f^{\prime}(z) / f(z)\right\}>0$ is the condition for a star mapping, which together with $f^{\prime}(0) \neq 0$ insures univalence.

Now let $G_{1}(z)=F(z) / z, G_{2}(z)=F^{\prime}(z), G_{3}(z)=z F^{\prime}(z) / F(z)$, and consider in general the majorizations

(A) $g_{1}(z)<G_{1}(z)$,

(B) $g_{2}(z)<G_{2}(z)$,

(C) $g_{3}(z)<G_{3}(z)$,

which were considered in $\$ 3.1$. Notice that if we let $f(z)=F(\alpha z) / \alpha$, where $|\alpha| \leqq 1$, then $g_{k}(z)=G_{k}(\alpha z)$ for $k=1,2,3$, and hence also $g_{k}(z)<G_{k}(z)$. On the other hand, as $\alpha$ varies, $g_{k}\left(z_{0}\right)$ assumes all values in the map of $|z| \leqq\left|z_{0}\right|$ by $G_{k}(z)$. Thus any one of the three majorizations, (A), (B), or (C), allows for $g_{k}\left(z_{0}\right)$ at least this set of values. Hence if from any one of the three majorizations, assumed in $|z|<1$, another one follows in $|z| \leqq r$, then in this circle it is certainly sharp, in the sense defined in the introduction. In par- 
ticular, all of the conclusions of the theorem of $\$ 3.1$ are sharp in the circles where they hold.

Suppose that from $g_{k}(z)<G_{k}(z)$ for $|z|<1$, it follows that $g_{l}(z)<G_{l}(z)$ for $|z| \leqq r$, but that $r$ cannot be replaced by a larger number. Then the problem of finding the possible values of $g_{l}\left(z_{0}\right)$ allowed by the hypothesis is solved for $\left|z_{0}\right| \leqq r$. If $\left|z_{0}\right|>r$, the problem is less simple. It is treated only incidentally in the following sections.

A final remark is the following. The function $g_{k}(z)$ is regular for $|z|<1$, and of the form $1+\cdots$, but is otherwise not at all special because it is of the form $f(z) / z, f^{\prime}(z)$, or $z f^{\prime}(z) / f(z)$. For each of the defining equations may be solved for $f(z)$ :$$
f(z)=z g_{1}(z),
$$$$
f(z)=\int_{0}^{z} g_{2}(\zeta) d \zeta
$$$$
f(z)=z \exp \int_{0}^{z}\left[g_{3}(\zeta)-1\right] \zeta^{-1} d \zeta .
$$

3.3. Problem AB. Here we are given

$$
\frac{f(z)}{z}<\frac{1+z}{1-z} \quad \text { for }|z|<1,
$$

and are to find a majorant for $f^{\prime}(z)$. To obtain this, we must apply to (A) the operator $\mathfrak{R}$ defined by $\mathfrak{g} g(z)=[z g(z)]^{\prime}$. We must verify that this is permissible.

First proof (using Part I). The operator $\mathfrak{Z}$ in question is $\mathfrak{Q}_{2} \mathfrak{P}_{1}$ of $\$ 1.2$, a linear operator of order zero. Hence by $\$ 1.3$, we may apply $\mathfrak{R}$ to (A) if we insert $\mathfrak{S}$ on the right, and the result is sharp. Thus we have

$$
f^{\prime}(z)<\mathfrak{S}\left\{\frac{2}{(1-z)^{2}}-1\right\} \quad \text { for }|z|<1 .
$$

This holds for $|z| \leqq r$ without the $\mathfrak{Q}$, if and only if the majorant is convex there, that is, if $h(z)=1 /(1-z)^{2}$ is convex. Now for convexity we must have

$$
\Re\left\{1+\frac{z h^{\prime \prime}(z)}{h^{\prime}(z)}\right\} \geqq 0
$$$$
\text { for }|z| \leqq r \text {, }
$$

that is, $\Re\{(1+2 z) /(1-z)\} \geqq 0$. This holds exactly in the circle having $-1 / 2$ and 1 as ends of a diameter, and hence for $|z| \leqq 1 / 2$ and in no larger circle about the origin.

Thus we have obtained the conclusion (AB) of the theorem of $\$ 3.1$. Actually, the result $\left(\mathrm{AB}^{*}\right)$ above is more complete, since it also shows what values are possible for $f^{\prime}\left(z_{0}\right)$ in case $\left|z_{0}\right|>1 / 2$.

Second proof (using Part II). We apply the theorem of $\$ 2.6$, with $G(z)$ $=(1+z) /(1-z)$. Notice that $G(z)$ is convex, and that $[z G(z)]^{\prime}=2 /(1-z)^{2}-1$ 
is univalent for $|z|<1$. Hence a necessary and sufficient condition that the desired conclusion

$$
f^{\prime}(z) \prec \frac{2}{(1-z)^{2}}-1
$$

is valid for $|z| \leqq r$ is that

$$
\left|\frac{2}{1-z}\right| \geqq \frac{2 r}{1-r^{2}} \quad \text { for }|z| \leqq r .
$$

This is correct if and only if $2 /(1+r) \geqq 2 r /\left(1-r^{2}\right)$, or $r \leqq 1 / 2$.

Remark. Recalling that $2 /(1-z)^{2}-1$ is convex for $|z| \leqq 1 / 2$, we see that either of the inequalities, $f^{\prime}(z) \neq 0$ or $\Re f^{\prime}(z)>0$, is always valid for $|z|<r$, if and only if $2 /(1+r)^{2}-1 \geqq 0$, or $r \leqq 2^{1 / 2}-1=0.414 \cdots$. In particular, we have the theorem: If $f(z)=z+\cdots$ is $r ?-n l$ lar for $|z|<1$, and $\Re\{f(z) / z\}>0$ there, then $f(z)$ is univalent for $|z| \leqq 2^{1 / 2}-: \cdot \cdot t$ not always in a larger circle about the origin.

3.4. Problem AC. Here again we are given (A), which is equivalent to

$$
\log \frac{f(z)}{z}<\log \frac{1+z}{1-z} \quad \text { for }|z|<1 .
$$

We are to find a majorant for $z f^{\prime}(z) / f(z)$. To obtain this, we must apply to $\left(\mathrm{A}^{\prime}\right)$ the operator $\mathfrak{R}$ defined by $\mathfrak{R} g(z)=z g^{\prime}(z)$, and then add 1 . We again give two proofs of the validity of this operation in a certain circle. The proof by the second method is given first, since it is simpler.

First proof (using Part II). We apply the theorem of $\$ 2.3$, with $G(z)$ $=\log [(1+z) /(1-z)]$. Since this function is convex, a necessary and sufficient condition that the desired conclusion

$$
\frac{z f^{\prime}(z)}{f(z)} \prec \frac{2 z}{1-z^{2}}+1
$$

is valid for $|z| \leqq r$ is that

$$
\left|\frac{1+z^{2}}{1-z^{2}}\right| \geqq \frac{2 r}{1-r^{2}} \quad \text { for }|z| \leqq r .
$$

This is true if and only if

$$
\frac{1-r^{2}}{1+r^{2}} \geqq \frac{2 r}{1-r^{2}},
$$

or $1-2 r-2 r^{2}-2 r^{3}+r^{4} \geqq 0$. The left side can be factored, giving

$$
\left[1-\left(1+5^{1 / 2}\right) r+r^{2}\right]\left[1-\left(1-5^{1 / 2}\right) r+r^{2}\right] \geqq 0 .
$$

Since the second factor is positive, we need only solve the first quadratic to find the required condition 


$$
r \leqq \frac{1+5^{1 / 2}}{2}-\left(\frac{1+5^{1 / 2}}{2}\right)^{1 / 2}=0.346 \cdots
$$

Second proof (using Part I). We have to apply to $\left(\mathrm{A}^{\prime}\right)$ the operator $\mathfrak{R}=\mathfrak{P}_{1} \mathfrak{Q}_{2}$ of $\$ 1.2$, a linear operator of order zero. Put

$$
L(z)=\mathfrak{R} \frac{1+z}{1-z}=\frac{2 z}{(1-z)^{2}},
$$

and let $C_{r}$ denote the map of $|z|=r$ by $L(z)$. Then, according to the theorem of $\$ 1.5$, a necessary and sufficient condition that (AC) holds for $|z| \leqq r$ is that for $|\zeta|=r$, the line joining $L(\zeta)$ and $L(-\zeta)$ doesn't cross $C_{r}$ again. If we assign to arcs of $C_{r}$ weights proportional to the lengths of the corresponding arcs of $|z|=r$, then this is equivalent to saying that in each direction there is a line which cuts $C_{r}$ into two pieces of equal weight.

Suppose that (AC) does not hold for $|z| \leqq r$. Then there is some line cutting $C_{r}$ into more than two pieces, such that the pieces on either side of the line have the same total weight. A parallel tangent to the curve can be found, such that the part of the curve on the side of the tangent indicated by the exterior normal to the curve has greater weight than the part on the other side. By varying the point of tangency continuously, we can find a tangent such that the part of the curve on either side has the same weight.

Let $|a|=|b|=1$. The tangent to $C_{r}$ at $L(a r)$ will pass through $L(b r)$ if and only if the quantity

$$
\frac{L(b r)-L(a r)}{i a L^{\prime}(a r)}=\frac{r(b-a)(1-a r)\left(1-a b r^{2}\right)}{i a(1+a r)(1-b r)^{2}}
$$

is real, and hence equal to its conjugate. This leads to the condition

$$
\frac{(1-a r)\left(1-a b r^{2}\right)}{(1+a r)(1-b r)^{2}}=\frac{(a-r)\left(a b-r^{2}\right)}{(a+r)(b-r)^{2}} \text {. }
$$

Clearing of fractions, and dividing out the factor $\left(1-r^{2}\right)(a-b)$, we have

$$
2 r^{2} a^{2} b^{2}-\left[r\left(1+r^{2}\right)\left(1+a^{2}\right)+a\left(1-r^{2}\right)^{2}\right] b+2 r^{2}=0 .
$$

The part of $C_{r}$ on either side of the tangent at $L(a r)$ has the same weight if and only if this equation is satisfied by two values of $b$ on the unit circle, one of which is the negative of the other. Thus the coefficient of $b$ must vanish, and in this case the roots $b= \pm i / a$ are on the unit circle. Hence the condition which $a$ must satisfy is

$$
r\left(1+r^{2}\right)\left(1+a^{2}\right)+a\left(1-r^{2}\right)^{2}=0 .
$$

This equation has two reciprocal solutions for $a$; they are either on the real axis or on the unit circle. We are interested in solutions with $|a|=1$, or 


$$
-2 \leqq a+a^{-1} \leqq 2 .
$$

Such solutions exist only if

$$
-2 r\left(1+r^{2}\right) \leqq\left(1-r^{2}\right)^{2} \leqq 2 r\left(1+r^{2}\right) .
$$

The left side always holds, but the right side holds only if

$$
1-2 r-2 r^{2}-2 r^{3}+r^{4} \leqq 0,
$$

or $r \geqq 0.346 \cdots$.

We have derived this inequality on the assumption that (AC) does not hold for $|z| \leqq r$. Thus (AC) holds at least for $|z|<0.346 \cdots$, and hence for $|z| \leqq 0.346 \cdots$.

On the other hand, the line joining $L( \pm i r)$ does cross $C_{r}$ again if

$$
\Re L(i r)<L(-r) \text {. }
$$

This condition reduces to $1-2 r-2 r^{2}-2 r^{3}+r^{4}<0$, or $r>0.346 \cdots$. Hence (AC) cannot hold for $|z| \leqq r$ if $r>0.346 \cdots$.

Remark. According to $\$ 1.5$, the conclusion (AC) holds in a circle whose radius is intermediate between the radii of convexity of the two functions

$$
\mathfrak{R} \frac{1+z}{1-z}=\frac{2 z}{(1-z)^{2}}, \quad \mathfrak{l} \log \frac{1+z}{1-z}=\frac{2 z}{1-z^{2}} .
$$

Simple calculations show that these radii are $2-3^{1 / 2}=0.267 \cdots$ (which is the smallest for any univalent function), and $2^{1 / 2}-1=0.414 \cdots$, respectively. The radius $0.346 \cdots$ which we found does lie between these.

3.5. Problem BA. We are given that

$$
f^{\prime}(z)<\frac{1+z}{1-z} \quad \text { for }|z|<1 .
$$

A majorant for $f(z) / z$ is desired. This may be obtained by applying the inverse of the operator $\mathfrak{R}$ for which $\mathfrak{R} g(z)=[z g(z)]^{\prime}$.

Because of the incompleteness of the results of $\$ 2.8$, which we would need to apply here, the second method can lead only to a partial result. Since a complete proof is obtained by the first method, we shall consider only this.

Proof (using Part I). The operator which we are to apply to (B) is $\mathfrak{Q}_{1} \mathfrak{P}_{2}$ of $\S 1.2$, which is a linear operator of order zero. By $\$ 1.3$, we can apply this operator, if we insert $\mathfrak{S}$ on the right. This gives

$$
\frac{f(z)}{z} \prec \mathfrak{S}\left\{\frac{2}{z} \log \frac{1}{1-z}-1\right\} \quad \text { for }|z|<1 .
$$

To complete the proof, we have only to show that the majorant is convex. The condition for convexity reduces to 


$$
\Re\left\{\left(\frac{z}{1-z}\right)^{2}:\left(\frac{z}{1-z}-\log \frac{1}{1-z}\right)\right\} \geqq 1 .
$$

Now $\Re w \geqq 1$ is equivalent to $|w-2| \leqq|w|$. Using this, the required inequality becomes

where

$$
|Q(z)| \leqq|P(z)|
$$

$$
P(z)=\left(\frac{z}{1-z}\right)^{2}, \quad Q(z)=P(z)-\frac{2 z}{1-z}+2 \log \frac{1}{1-z} .
$$

We find that

$$
P^{\prime}(z)=2 z /(1-z)^{3}, \quad Q^{\prime}(z)=2 z^{2} /(1-z)^{3},
$$

and hence

$$
\left|Q^{\prime}(z)\right| \leqq\left|P^{\prime}(z)\right|
$$

Notice that the transformation $w=P(z)$ can be obtained by setting

$$
\omega=z /(1-z), \quad w=\omega^{2} .
$$

The first transformation takes $|z|<1$ into the half-plane $\Re \omega>-1 / 2$, which is star-shaped with respect to the origin; the second transformation takes this half-plane into a two-sheeted region, which is also star-shaped. Thus the desired conclusion can be obtained from the following lemma.

Lemma. If $P(z)$ and $Q(z)$ are regular for $|z|<1$,

$$
\left|Q^{\prime}(z)\right| \leqq\left|P^{\prime}(z)\right| \quad \text { for }|z|<1 \text {, }
$$

$Q(0)=P(0)=0$, and if $P(z)$ maps $|z|<1$ onto a (many-sheeted) region which is star-shaped with respect to the origin, then

$$
|Q(z)| \leqq|P(z)| \quad \text { for }|z|<1 .
$$

Proof. Suppose that $z_{0}$ is given, with $\left|z_{0}\right|<1$. Join 0 to $P\left(z_{0}\right)$ by a line segment. This segment lies in the map of $|z|<1$ by $P(z)$, and hence is the map of a curve $C$, lying in the unit circle, and joining 0 to $z_{0}$. Then we have

$$
\left|Q\left(z_{0}\right)\right| \leqq \int_{C}\left|Q^{\prime}(\zeta)\right||d \zeta| \leqq \int_{C}\left|P^{\prime}(\zeta)\right||d \zeta|=P\left(z_{0}\right) .
$$

Remarks. The condition about star mapping is equivalent to assuming that $P(z)$ is a power of a univalent function which defines a star mapping. If this condition were omitted, the lemma would be incorrect. For if we put

$$
P(z)=500\left(e^{8 z}-1\right), \quad Q(z)=z,
$$

then we have $\left|P^{\prime}(z)\right|>1=Q^{\prime}(z)$ in the unit circle, but $P(z)$ has zeros in $0<|z|<1$, so that the conclusion cannot hold.

3.6. Problem BC. Here again we are given (B), but we shall use only 
(BA), which was shown in $\S 3.5$ to be a consequence of (B). Since the majorant in (BA) is the average of $(1+\zeta) /(1-\zeta)$ on the segment joining 0 and $z$, it certainly has a positive real part, so that its logarithm is regular. Thus (BA) is equivalent to

$$
\log \frac{f(z)}{z}<\log \left(\frac{2}{z} \log \frac{1}{1-z}-1\right) \quad \text { for }|z|<1 .
$$

A majorant for $z f^{\prime}(z) / f(z)$ is required. This may be obtained by applying to $\left(\mathrm{BA}^{\prime}\right)$ the operator $\mathfrak{l}$ defined by $\mathfrak{l} g(z)=z g^{\prime}(z)$, and then adding 1 . Since the majorant in $\left(\mathrm{BA}^{\prime}\right)$ was not considered in Part $\mathrm{I}$, we have only the second method to use to show that we may apply this operator.

Proof (using Part II). Because of the complicated nature of the majorant in $\left(\mathrm{BA}^{\prime}\right)$, we shall not attempt to determine the largest radius for which we can apply the operator $\mathbb{R}$. Without looking at the majorant, we see by $\$ 2.4$ that this is permissible for $|z| \leqq 3-2^{3 / 2}=0.171 \cdots$. However, we shall make a better estimate than this, by applying the theorem of $\$ 2.3$ with

$$
G(z)=\log \left(\frac{2}{z} \log \frac{1}{1-z}-1\right) .
$$

We can apply the operator $\mathfrak{R}$, obtaining the conclusion

$$
\frac{z f^{\prime}(z)}{f(z)} \prec \frac{1+z}{1-z}:\left(\frac{2}{z} \log \frac{1}{1-z}-1\right)
$$

for $|z| \leqq r$, provided that

$$
\Re\left\{1+\frac{z G^{\prime \prime}(z)}{G^{\prime}(z)}\right\} \geqq 0 \text { and }\left|1+\frac{z G^{\prime \prime}(z)}{G^{\prime}(z)}\right| \geqq \frac{2 r}{1-r^{2}}
$$

for $|z| \leqq r$. Now

$$
1+\frac{z G^{\prime \prime}(z)}{G^{\prime}(z)}=A(z)-B(z)
$$

where

$$
\begin{aligned}
& A(z)=\frac{z}{1-z}:\left(1-\frac{1-z}{z} \log \frac{1}{1-z}\right)=\sum_{n=1}^{\infty} z^{n}: \sum_{n=1}^{\infty} \frac{z^{n}}{n(n+1)}, \\
& B(z)=\frac{1+z}{1-z}:\left(\frac{2}{z} \log \frac{1}{1-z}-1\right)=\left(1+2 \sum_{n=1}^{\infty} z^{n}\right):\left(1+2 \sum_{n=1}^{\infty} \frac{z^{n}}{n+1}\right) .
\end{aligned}
$$

Thus both $A(z)$ and $B(z)$ are expressed as quotients of power series with positive coefficients; in the numerators the coefficients are nondecreasing, and in the denominators nonincreasing. Hence $A(z)$ and $B(z)$ have series expansions with positive coefficients: $A(z)=2+(4 / 3) z+\cdots, B(z)=1+z+\cdots$. Thus $A(z)-2-z$ and $B(z)-1-z$ also have positive coefficients, so that 


$$
|A(z)-2-z| \leqq A(r)-2-r, \quad|B(z)-1-z| \leqq B(r)-1-r,
$$

and hence $|A(z)-B(z)-1| \leqq A(r)+B(r)-3-2 r$, for $|z| \leqq r$. The two required conditions

$$
\Re\{A(z)-B(z)\} \geqq 0, \quad|A(z)-B(z)| \geqq 2 r /\left(1-r^{2}\right)
$$

are certainly both satisfied if

$$
|A(z)-B(z)-1| \leqq 1-2 r /\left(1-r^{2}\right)
$$

for $|z| \leqq r$, which is true if

$$
A(r)+B(r)-3-2 r \leqq 1-2 r /\left(1-r^{2}\right) .
$$

This is found to be correct for $r=0.3$.

3.7. Problem $\mathrm{CA}$. We are given

$$
\frac{z f^{\prime}(z)}{f(z)}<\frac{1+z}{1-z} \quad \text { for }|z|<1 .
$$

In other words, we assume that $f(z)$ maps $|z|<1$ onto a star-shaped region. A majorant for $f(z) / z$ is desired. If we subtract 1 from (C), and then apply the inverse of the operator $\mathfrak{l}$ for which $\mathfrak{l} g(z)=z g^{\prime}(z)$, we obtain a majorant for $\log [f(z) / z]$.

Because of the incompleteness of the results of $\$ 2.8$, which we would need to apply here, the second method can lead only to a partial result. Since a complete proof is obtained by the first method, we shall consider only this.

Proof (using Part I). The operator which we are to apply to (C) is $\mathfrak{B}_{2} \mathfrak{Q}_{1}$ of $\$ 1.2$, which is a linear operator of order zero. By $\$ 1.3$, we can apply this operator, if we insert $\mathfrak{S}$ on the right. This gives

$$
\log \frac{f(z)}{z} \prec \mathfrak{Q}\left\{2 \log \frac{1}{1-z}\right\}
$$$$
\text { for }|z|<1 \text {. }
$$

The condition that the majorant be convex reduces to $\Re\{1 /(1-z)\} \geqq 0$, which is true; hence the $\mathfrak{S}$ can be omitted. We may then also omit the logarithm, obtaining

$$
\frac{f(z)}{z}<\frac{1}{(1-z)^{2}}
$$$$
\text { for }|z|<1 \text {, }
$$

since the new majorant is univalent. Notice that this determines the set of possible values of $f\left(z_{0}\right)$ for any fixed $z_{0}$ and all star mappings. The extremal function is $f(z)=z /(1-\alpha z)^{2}$ with $|\alpha|=1$, which maps $|z|<1$ onto a plane with a radial slit $\left({ }^{13}\right)$.

3.8. Problem $\mathrm{CB}$. Here again we are given (C), but we shall use only (CA),

(13) Marx, paper cited in footnote 5, Satz B. 
which was shown in $\S 3.7$ to be a consequence of $(C)$. The desired majorant for $f^{\prime}(z)$ can be obtained by applying to (CA) the operator $\&$ defined by $\mathfrak{R} g(z)$ $=[z g(z)]^{\prime}$. Two proofs will be given that this is possible in some circle.

First proof (using Part I). The operator $\mathfrak{R}$ is $\mathfrak{Q}_{2} \mathfrak{P}_{1}$ of $\S 1.2$, a linear operator of order zero. By $\$ 1.4$; we can apply this operator to $(C A)$, if we insert $\mathfrak{W}$ on the right. This gives

$$
f^{\prime}(z)<\mathfrak{E}\left\{\frac{1+z}{(1-z)^{3}}\right\} \quad \text { for }|z|<1 ;
$$

but this estimate is not known to be sharp even under the hypothesis (CA). From $\left(\mathrm{CB}^{*}\right)$, we can obtain $(\mathrm{CB})$ for $|z| \leqq r$ only if the majorant is convex there. The convexity condition reduces to

$$
\Re \frac{1+4 z+z^{2}}{(1-z)(2+z)} \geqq 0 .
$$

For $z=-r$, this is evidently true if and only if $r \leqq 2-3^{1 / 2}=0.267 \cdots$, and we shall show that this determines the radius of convexity. In fact, the required condition is equivalent to

$$
\Re\left\{\left(1+4 z+z^{2}\right)(1-\bar{z})(2+\bar{z})\right\} \geqq 0 .
$$

If we let $r=|z|$ and $x=\Re z$, this becomes

$$
\left(2-5 r^{2}-r^{4}\right)+\left(7-5 r^{2}\right) x+2 x^{2} \geqq 0 .
$$

The derivative of the left side with respect to $x$ is $7-5 r^{2}+4 x$, which is positive at least for $r \leqq 1 / 2$, so that the critical case is $x=-r$, or $z=-r$.

From $\left(\mathrm{CB}^{*}\right)$ we can obtain (CB) only for $|z| \leqq 2-3^{1 / 2}$; the larger radius given in the theorem of $\$ 3.1$ will be found in the second proof. On the other hand, $\left(\mathrm{CB}^{*}\right)$ does give some information not given by that theorem.

Second proof (using Part II). We apply the theorem of $\$ 2.6$, with $G(z)$ $=1 /(1-z)^{2}$. This function is convex for $|z| \leqq 1 / 2$, as was already noted in §3.3. We must also check the univalence of

$$
H(z)=[z G(z)]^{\prime}=\frac{1+z}{(1-z)^{8}} .
$$

First we shall show that $\log H(z)$ is univalent; it is sufficient to show that its derivative has a positive real part. Now

$$
[\log H(z)]^{\prime}=(4+2 z) /\left(1-z^{2}\right),
$$

and, putting $r=|z|$, the real part of this is, except for a positive factor, $\Re\left\{(2+z)\left(1-\bar{z}^{2}\right)\right\}=2 \Re\left\{1-z^{2}\right\}+\left(1-r^{2}\right) \Re z \geqq 2\left(1-r^{2}\right)-\left(1-r^{2}\right) r>0$. Thus $\log H(z)$ is univalent. Also, 


$$
|\operatorname{amp} H(z)|<4 \text { arc sin } r \text {, }
$$

so that $H(z)$ is not negative for $|z| \leqq 2^{-1 / 2}$, and hence is univalent there.

Having settled these preliminaries, we see that the desired conclusion

$$
f^{\prime}(z) \prec \frac{1+z}{(1-z)^{8}}
$$

for $|z| \leqq r$, where $r \leqq 1 / 2$, will follow from (CA) if and only if

$$
\left|\frac{2+z}{1-z}\right| \geqq \frac{2 r}{1-r^{2}} \quad \text { for }|z| \leqq r .
$$

This is true provided $(2-r) /(1+r) \geqq 2 r /\left(1-r^{2}\right)$, or $r \leqq\left(5-17^{1 / 2}\right) / 2=0.438 \cdots$. This is the largest radius for which (CB) follows from (CA), but not necessarily the largest radius for which (CB) follows from (C). Notice that this radius is larger than the radius of convexity of the majorant.

Remarks. Marx has conjectured that in the whole unit circle, $f(z)$ $=z /(1-z)^{2}$ serves as an extremal function so far as values of $f^{\prime}(z)$ for star mappings are concerned $\left({ }^{14}\right)$. We cannot say that he conjectured $(\mathrm{CB})$ for $|z|<1$, since by our definition this fails because the majorant is not univalent. His conjecture could be written

$$
\log f^{\prime}(z) \prec \log \frac{1+z}{(1-z)^{3}}
$$

for $|z|<1$. As a contribution to this conjecture, he proved $\left(\mathrm{CB}^{*}\right)$, by a method related to our first proof, and from this $(\mathrm{CB})$ or $\left(\mathrm{CB}^{\prime}\right)$ follows for $|z| \leqq 2-3^{1 / 2}=0.267 \cdots$. We have increased the radius of the circle where (CB) is known to be true to $\left(5-17^{1 / 2}\right) / 2=0.438 \cdots$, using a quite different method, but still making the result depend on (CA). Any further increase requires that $(\mathrm{CB})$ be deduced from $(C)$ itself, and not from (CA).

3.9. In \$3.6, we have drawn conclusions about (BC) from (BA) rather than from (B), and in $\$ 3.8$ conclusions about (CB) from (CA) rather than from $(C)$. We have done this since there was no apparent way of connecting (B) and (C) except via (A). That this procedure can hardly be expected to give a maximum radius is made clear by the following example, which is also interesting in itself.

Starting from (C), we find (CA), and this is sharp. But from (CA), can we recover $(\mathrm{C})$ ? Writing $(\mathrm{CA})$ in the equivalent form

$$
\log \frac{f(z)}{z}<2 \log \frac{1}{1-z} \quad \text { for }|z|<1,
$$

we can apply the theorem of $\S 2.3$, with $G(z)=2 \log [1 /(1-z)]$. As noted

(14) Marx, loc. cit. p. 66. 
in $\$ 3.7, G(z)$ is convex. Hence a necessary and sufficient condition that the conclusion

$$
\frac{z f^{\prime}(z)}{f(z)} \prec \frac{1+z}{1-z}
$$

for $|z| \leqq r$ will follow, is that

$$
\left|\frac{1}{1-z}\right| \geqq \frac{2 r}{1-r^{2}} \quad \text { for }|z| \leqq r .
$$

This is true only if $1 /(1+r) \geqq 2 r /\left(1-r^{2}\right)$, or $r \leqq 1 / 3$. Thus from (CA) for $|z|<1$ follows (C) only for $|z| \leqq 1 / 3$, despite the fact that, assuming (C), (CA) gives a sharp majorant for $f(z) / z$.

We thus have the following result: If $f(z)=z+\cdots$ is regular for $|z|<1$, and does not at any point assume a value which would be impossible if $f(z)$ defined a star mapping of the unit circle, then $f(z)$ does in fact define a star mapping for $|z| \leqq 1 / 3$. The number $1 / 3$ cannot be replaced by any larger constant.

UNIVERSITY OF CALIForNia, Berkeley, Calif. 